UCRL-ID-127002

?

\title{
Theoretical Issues in Spheromak Research
}

R.H. Cohen, E.B. Hooper, L.L. LoDestro, N. Mattor, L.D. Pearlstein, D.D. Ryutov

April 1, 1997

This is an informal report intended primarily for internal or limited external distribution. The opinions and conclusions stated are those of the author and may or may not be those of the Laboratory.

Work performed under the auspices of the Department of Energy by the Lawrence Livermore National Laboratory under Contract W-7405-Eng-48. 


\section{DISCLAIMER}

This document was prepared as an account of work sponsored by an agency of the United States Government. Neither the United States Government nor the University of California nor any of their employees, makes any warranty, express or implied, or assumes any legal liability or responsibility for the accuracy, completeness, or usefulness of any information, apparatus, product, or process disclosed, or represents that its use would not infringe privately owned rights. Reference herein to any specific commercial product, process, or service by trade name, trademark, manufacturer, or otherwise, does not necessarily constitute or imply its endorsement, recommendation, or favoring by the United States Government or the University of Califomia. The views and opinions of authors expressed herein do not necessarily state or reflect those of the United States Government or the University of Califomia, and shall not be used for advertising or product endorsement purposes.

This report has been reproduced directly from the best available copy.

Available to DOE and DOE contractors from the Office of Scientific and Technical Information P.O. Box 62, Oak Ridge, TN 37831

Prices available from (615) 576-8401, FTS 626-8401

Available to the public from the

National Technical Information Service.

U.S. Department of Commerce

5285 Port Royal Rd.

Springfield, VA 22161 


\section{Theoretical Issues in Spheromak Research}

R. H. Cohen, E. B. Hooper, L. L. LoDestro, N. Mattor, L. D. Pearlstein, D. D. Ryutov

Lawrence Livermore National Laboratory

P. O. Box 808

Livermore, CA 94551-9900 


\section{Contents}

$\begin{array}{ll}\text { 1. Introduction } & 1.1\end{array}$

2. MHD Equilibrium and Design 2.1

2.1 MHD equilibrium 2.1

2.2 Mercier limits 2.3

References $\quad 2.6$

3. Review of the Stability of the Spheromak 3.1

3.1 Low- $n$ modes 3.3

3.2 High-n modes; Mercier condition $\quad 3.10$

3.3 Resistive modes $\quad 3.12$

3.4 MHD issues remaining to be addressed 3.15

References $\quad 3.17$

4. Spheromak Dynamo 4.1

4.1 Basic description of the dynamo in relaxed-state devices 4.1

4.2 Dynamo in a spheromak plasma 4.3

4.3 Proposed course of study for the spheromak dynamo $\quad 4.5$

References $\quad 4.10$

5. Edge Plasma in Spheromaks $\quad 5.1$

5.1 Introduction 5.1

5.2 The role of skin effect in the flux conserver 5.1

5.3 Plasma collisionality $\quad 5.4$

5.4 Current flow on the open field lines $\quad 5.4$

5.5 Longitudinal pressure equilibrium 5.5

5.6 The thermal equilibrium 5.5

5.7 Radiative losses $\quad 5.7$

5.8 Plasma resistivity $\quad 5.9$

5.9 Heat flux through the separatrix $\quad 5.10$

5.10 Particle balance (ionization equilibrium) $\quad 5.10$

5.11 Generation of impurities $\quad 5.11$

5.12 Issues for further study $\quad 5.12$

References $\quad 5.12$ 


\section{Introduction}

A fusion reactor based on the spheromak potentially escapes many of the issues associated with the tokamak, especially those due to the presence of a central toroidal magnet coil and the central solenoid used for inductive current drive. By eliminating these a potentially more compact and simpler reactor results.

The spheromak, however, has much "richer" physics than the tokamak. The current profile in a spheromak is subject to relaxation processes. These processes drive the discharge current, but at the cost of opening magnetic field lines and reducing energy confinement. Although extrapolation of these processes from previous experimental results to reactor conditions is promising, the considerations are based on relatively few experimental results, especially for high-temperature spheromaks sustained by steady-state (time-averaged) helicity injection.

Addressing confinement and other physics in the spheromak requires both experimental and theoretical exploration. Analysis of important issues will:

- Allow us to determine how to operate the spheromak with a plasma of fusion relevance. We will study important spheromak physics issues, especially those which impede progress towards the reactor regime. By understanding such constraints we can search for changes in the basic spheromak geometry and operation to improve the energy confinement, stability, and beta limits.

- Provide the knowledge and tools to design an advanced spheromak experiment ("proofof-principle") which can move into the long pulse, fusion parameter regime.

- Advance plasma physics in several important domains. Particularly interesting is improved understanding of the magnetic dynamo in high temperature (low resistivity) plasmas. The dynamo plays a major role in the generation of magnetic fields in space and in the sun and earth. Although the dominant physical parameters differ vastly. between these natural settings and the laboratory, a better understanding of the processes in the lab will advance a broader understanding. In this sense, the spheromak is complementary to the reversed field pinch (RFP); they have closely related physics but with sufficient difference in the geometry and current profiles that we expect to gain physics understanding by comparing results from both devices.

This report summarizes the state of theoretical knowledge of several physics issues important to the spheromak. It was prepared as part of the preparation for the Sustained Spheromak Physics Experiment (SSPX), which has goals to address: 
- Energy confinement and the physics which determines it. Can good energy confinement be obtained in a spheromak subject to the magnetic dynamo, or will current profile control be required? What determines the beta limit in practice, and how can it be maximized subject to other operational constraints? These issues can be addressed economically in a short-pulsed experiment.

- The physics of transition from a short-pulsed experiment, in which the equilibrium and stability are determined by a conducting wall ("flux conserver") to one in which the equilibrium is supported by external coils. The dominant, low order MHD modes will become resistive-wall modes on the long timescale, and feedback or other stabilization techniques are required. It is anticipated that the SSPX will use a resistive wall (in a second phase of experiments) to allow exploration of these processes on a relatively short timescale $(\sim 10 \mathrm{~ms})$.

Physics is examined in this report in four important areas. The status of present theoretical understanding is reviewed, physics which needs to be addressed more fully is identified, and tools which are available or require development are described. Specifically, the topics include:

MHD Equilibrium and Design. Calculation of MHD equilibria for the spheromak is a mature subject with no major new development required. Modern computational tools allow very rapid calculations to be performed, permitting much more rapid evaluation of new ideas and geometries than previously possible. The chapter on equilibrium includes a discussion of the equilibrium and an example. Also included are calculations of the Mercier stability limit for the confined plasma, including the case in which current flows on the open field lines surrounding the plasma. It is found that the resultant toroidal magnetic field reduces the shear, and thus the Mercier limit, unless the current profile in the spheromak is shaped carefully. The issue of the effect of external current is also considered in the chapter on MHD stability.

Review of MHD Stability. We review and comment on extensive previous calculations of MHD stability in the spheromak. These calculations provide a basis for evaluating the regimes stable to both current and pressure driven modes, in terms of the geometry and of current and pressure profiles. Several issues which require further work are described, including the effects of current on open magnetic flux surfaces. The development of computational tools to analyze feedback control of low m,n MHD modes is discussed. 
Spheromak Dynamo. The dynamo is important to, and couples together a wide range of physics in the spheromak. Current understanding of the dynamo is discussed, including the similarities and differences with the RFP. As understanding of the physics is limited, this area offers fruitful opportunities for study. To limit energy losses, it may prove necessary to stabilize the dynamo modes, by direct feedback or control of the current profile; several possibilities are identified.

Edge Plasma in Spheromaks. The edge plasma in the spheromak plays a major role in experiment: Current densities are high, wall interactions are important, and the coupling of the dynamo drive with the helicity injector involves electrodes or other walls. We determine the dominant issues by order-of-magnitude estimates. Included are the effects of field lines which contact walls because of field errors or flux diffusion, consequences of the current flow, energy losses, atomic processes, and particle balance. Although the longterm goal is the application of computational analysis to handle the multiple physics issues self-consistently, there are numerous issues which can be analyzed at a lower level of effort. These include plasma-electrode interactions in the helicity injector, impurity entrainment, and line-tying effects among others. Our long-term goal for the theoretical study of edge physics is to apply capabilities developed in recent years because of the importance of the edge in the tokamak. These capabilities, which include both analysis and computation, offer opportunities to understand and optimize the edge and its interactions with walls and electrodes.

This work was performed under the auspices of the U.S. Department of Energy by the Lawrence Livermore National Laboratory under Contract W-7405-ENG-48. 


\section{MHD Equilibrium and Design}

\subsection{MHD equilibrium}

The ideal MHD equilibrium of the spheromak is conveniently described by the parameter $\lambda(\psi)=d F / d \psi$, with $\psi$ the poloidal flux and $F(\psi)=R B_{\text {. }}$. (In RFP and some spheromak literature $\lambda$ is termed $\mu$.) At low beta, this becomes

$$
\lambda=\frac{\mu_{0} \mathbf{j} \bullet \mathbf{B}}{B^{2}} .
$$

More generally, inclusion of the diamagnetic current yields

$$
\frac{d F}{d \psi}=\frac{\mu_{0}}{B^{2}}\left(\mathbf{j} \cdot \mathbf{B}-\frac{d p}{d \psi} F\right)
$$

We use this in the Grad-Shafranov equation:

$$
\Delta^{*} \psi=-\lambda \int_{0}^{\psi} \lambda d \psi-\mu_{0} r^{2} \frac{d p}{d \psi}
$$

where

$$
\Delta^{*} \equiv r \frac{\partial}{\partial r} \frac{1}{r} \frac{\partial}{\partial r}+\frac{\partial^{2}}{\partial z^{2}}
$$

The limit $\lambda=$ constant and $p=0$ describes the Taylor minimum energy state.'

The Grad-Shafranov equation is known to be an eigenvalue equation despite its nonlinear character. ${ }^{2}$ The equilibrium geometry can be determined either by external coils or by a flux-conserving (conducting) boundary. Analysis of the solutions to this equation in a flux conserver (but with helicity injected across part of the boundary) has been carried out by Kitson and Browning, ${ }^{3}$ who generate equilibria with an x-point for the SPHEX experiment. LoDestro and Pearlstein ${ }^{4}$ discuss the implications of this eigenvalue character for the Grad-Shafranov equation when expressed in terms of the safety factor, $q(\psi)$.

We have used the Corsica code to determine equilibria of interest for the SSPX. For the cases described here, the flux conserver is modeled by a large number of small coils, and the zero-flux boundary, which is outside the separatrix, lies on these coils. A design consideration is to have the current which flows in the wall smoothly varying so as to minimize the differential leakage of flux into the metal. The basic geometry for the design is based on several considerations; final design will evolve from this starting point:

- The CTX experiment at Los Alamos achieved its highest temperature results (in a decaying plasma) for a cylindrical flux conserver with the outer radius of the coaxial 
gun approximately equal to the flux conserver radius. ${ }^{5}$ The coaxial gap is designed so that in the gun $\lambda_{g} \approx \pi /($ gap width $)>\lambda$.

- Estimates of the energy required to buildup the magnetic field (including an efficiency factor) when compared to the energy available from the capacitor bank planned for the experiment indicate that the major radius, $R$, of the flux conserver should be no larger than about $0.4 \mathrm{~m}^{6}$

- There have been extensive calculations of the tilt/shift mode stability in spheromaks, which we use to guide our design. In the original spherical geometry with a conducting wall on the plasma surface, stability was achieved by making the wall prolate. ${ }^{7}$ Similar calculations for cylindrical flux conservers showed that $L R$, with $L$ the cylinder length, needs to be $<1.67 .^{8}$ Analysis by Jardin' of geometries with flux holes along the geometric axis and variable elongation yield stability conditions which are sensitive to the distance between the plasma boundary and the conducting wall. Based on these results and previous experiments, we choose $L R=1.25$ as the base design case for our geometry, which is approximately cylindrical.

- To minimize the effects of flux penetration into corners, we round the bottom of the flux conserver and the inner cylinder of the coaxial gun.

A typical equilibrium is shown in Fig. 1. The Taylor state with $\lambda=$ constant, $p=0$, and no current flowing on external fieldlines is shown in this first example; here $\lambda=0.145$ $\mathrm{cm}^{-1}$. Figures $2-4$ show several features of the spheromak equilibrium. In these and subsequent plots, $\psi$ is normalized to vary from 0 on the magnetic axis to 1 at the separatrix.

This equilibrium was tested for low-m,n mode stability using the GATO code, and found to be stable in the presence of the conducting wall. ${ }^{10}$ In the absence of a conducting wall, the equilibrium is unstable to tilt.

Adding current on the open fieldlines (inside the zero-flux surface) has only minor effects on the separatrix geometry as can be seen in Fig. 5. In this case, the profile of $\lambda$ has been taken constant within the zero-flux surface, and its value has decreased to 0.116 $\mathrm{cm}^{-1}$ because of the external current. For the case shown, the toroidal current within the last closed flux surface $=1 \mathrm{MA}$ and the external current $=0.30 \mathrm{MA}$, corresponding to a toroidal current amplification factor of 3.5. 
The primary manifestations of external current are seen in $|B|$ (Figs. 6-7), where the field maximum is now on the geometric axis, and in the profile of $q$ (Fig. 8). The logarithmic divergence of the safety factor on the separatrix, not fully resolved in this plot, results from the nonzero toroidal field due to the external current.

The magnitude of the external current is proportional to the open flux as we have chosen $\lambda=$ constant between the separatrix and the geometric axis. This flux is generated by the plasma gun solenoids. Figures. 9-12 show the geometry and spheromak parameters for a current amplification of 7.2; we still find $\lambda=0.116 \mathrm{~cm}^{-1}$. We see several minor differences: the separatrix extends closer to the geometric axis; $\mathbb{B l}$ is slightly smaller; and the value of $q_{\min }$ is smaller; but otherwise, the features are similar to the previous case.

The actual current amplification which can be achieved in the experiment is determined by helicity and energy balances, and thus is not evaluated within ideal MHD theory. Determination of the optimized shape of the flux conserver will be dependent on these and other considerations which lie outside the present discussion.

\subsection{Mercier limits}

Previous calculations at the low magnetic shear of the force-free, Taylor state have found low beta limits $(-1 \%)$ at the Mercier limit. Experiments, however, often reached higher beta. ${ }^{11}$ The reasons are not understood; see Jarboe ${ }^{12}$ for discussion of possibilities such as deviations in the current profile from the Taylor state, which permits betas of several tens of percents, or from effects such as shear of the plasma flow which lie outside the model.

The results described below extend previous calculations of the beta limits in spheromaks ${ }^{27,13-16}$ to include a geometry which couples to a plasma gun or other external helicity injector. We differ from previous work by including a separatrix and, in some of our work, current flowing on external (open) field lines. The earlier studies usually analyzed an equilibrium with magnetic field geometry similar to that of the classic spheromak $^{7}$ with point separatrices on the geometric axis, or of a plasma confined within a flux surface which had a flux hole along the geometric axis. Experimentally, this would presumably have required a toroidal flux conserver or a limiter. The plasmas were characterized by ellipticity and an aspect ratio. At high aspect ratios and with $q_{0}=1$, very high betas were found; for example, Gautier, et al. ${ }^{2}$ found $\beta_{\max }$ varied from 0.015 for no hole to 0.40 for an aspect ratio of 2.34 . 
The spheromak relies on shear to stabilize the internal interchange modes, and we use the Mercier condition to determine the pressure profile. For a given geometry, the magnitude of the resulting beta will be determined primarily by $q_{0}$, by the effects of currents flowing in the flux hole along the geometric axis, and by shear due to the profile in $\lambda$. This latter consideration is particularly important for a spheromak sustained by helicity injection; the associated external current geometry is constrained if the goal is high beta.

The safety factor can be written as

$$
q(\psi)=\frac{F(\psi)}{2 \pi} \oint \frac{d \ell}{R^{2} B_{p}}
$$

The value of $q$ on the separatrix is quite sensitive to the boundary conditions. In the original MHD calculations" on the "classical spheromak," it was noted that in the absence of a flux hole along the geometric axis the value of $q$ on the separatrix was nonzero, but that even a small flux hole will make $q(1)=0$ if $F(1)=0$. However, any currents flowing on the open flux along the geometric axis will generate a toroidal magnetic field on the separatrix, so that $F(1) \neq 0$. In this case, the safety factor diverges as in the tokamak, rather than dropping to zero. We will see that this has a significant impact on the shear throughout the volume, often resulting in a significantly lower Mercier-stable beta than in the absence of the current. An interesting option is to prevent this current from flowing, but this would require a different mechanism for coupling to the interior dynamo than usually observed in experiments. Alternatively, an auxiliary current drive might be used.

To first approximation, the shear is proportional to the difference between the safety factor on axis and its minimum near the edge of the confinement volume. The safety factor on the magnetic axis for surfaces of ellipticity $\kappa_{0}$ can be shown to be

$$
q_{0}=\frac{2}{\lambda_{0} R_{0}} \frac{1+\kappa_{0}^{2}}{2 \kappa_{0}}
$$

where $R_{o}$ is the radius at the magnetic axis. For the case in which there is a flux hole without current through it, so at the edge $q=0$, we will find that the maximum Mercierstable $\beta_{p}$ is proportional to $q_{0}$. As seen from Figs. 8 and 12 , the situation is more complex when there is current through the hole, with a minimum of $q$ near the separatrix which is nonzero.

We expand $\lambda$ in flux:

$$
\lambda(\psi)=\text { const } *\left(1+a_{1} \psi^{1}+a_{2} \psi^{2}+a_{3} \psi^{3}+\ldots\right) .
$$


The constant is determined as part of the equilibrium calculation. Terms through the cubic are included in these calculations; inclusion of higher powers might improve the optimizations. The maximum beta is characterized by $\beta_{p}$, defined as the volume-averaged pressure divided by the surface-averaged, squared poloidal magnetic field:

$$
\beta_{p}=2 \mu_{0}\langle p\rangle_{v o l} /\left\langle B_{p}^{2}\right\rangle_{\text {suface }} .
$$

The pressure is required to match the Mercier condition at all radii except at the edge where we set $d p / d \psi=0$.

Beta will be maximized without considering most other physics constraints, but we will require $q_{o}<1$ and (for this initial set of calculations) $\partial \lambda / \partial \psi \geq 0$ throughout the profile. Helicity injection is generally thought to occur via tearing modes, and there is experimental evidence ${ }^{17}$ that during sustainment by helicity injection the positive $\lambda$ gradient condition is satisfied on the average (at least) throughout the plasma. This is sometimes stated in the literature as "Helicity flows downhill in $\lambda$. ." The impact of the current profile on physics other than this will be neglected here.

For monotonic $\lambda$, the maximum stable $\beta_{p}$ depends strongly on $q_{0}$ and only weakly on the precise gradient in $\lambda$, although the precise pressure distribution does depend on this gradient. Write $\lambda$ as

$$
\frac{\lambda}{\lambda_{0}}=1+\lambda_{0}^{\prime} \psi+\frac{1}{2}\left(\lambda_{1}^{\prime}-\lambda_{0}^{\prime}-3 a_{3}\right) \psi^{2}+a_{3} \psi^{3}
$$

where $\lambda_{0}^{\prime}$ and $\lambda_{1}^{\prime}$ are the normalized derivatives at $\psi=0$ and 1 , respectively, both $\geq 0$ to satisfy the assumed monotonic increase of $\lambda$ with flux. The derivative of $\lambda$ is thus

$$
\lambda^{\prime} / \lambda_{0}=\lambda_{0}^{\prime}+\left(\lambda_{1}^{\prime}-\lambda_{0}^{\prime}-3 a_{3}\right) \psi+3 a_{3} \psi^{2} .
$$

The condition for $\lambda^{\prime} \geq 0$ in the range $0 \leq \psi \leq 1$ is $\lambda_{0}^{\prime} \geq 0, \lambda_{1}^{\prime} \geq 0$, and

$$
a_{3} \leq\left(\sqrt{\lambda_{0}^{\prime}}+\sqrt{\lambda_{1}^{\prime}}\right)^{2} / 3
$$

Numerically, we find that the maximum beta occurs at or very close to $\lambda_{0}^{\prime}=\lambda_{1}^{\prime}=0$, and thus with $a_{3} \leq 0$. This places the gradient of $\lambda$ where the pressure gradient is largest, as $d p / d \psi=0$ on the magnetic axis (as required by the Mercier condition) and $p$ is small near 
the separatrix. Thus, we take $a_{1}=0$ and $a_{2}=-2 a_{3} / 3$. Results are shown in Fig. 13; the configuration has sufficient shear to support a Mercier stable $\beta_{p} \approx 0.3$ at $q(0)=1.0$.

When we examine the Mercier limit in cases with current through the flux hole along the geometric axis, we find that the divergence in $q$ on the separatrix results in an appreciable increase in $q_{\min }$ so that there is less increase in shear than one might anticipate. The result is a Mercier limited $\beta_{p}$ which is reduced by an order-of-magnitude from the current free case, as seen in the lower curve in Fig. 14. To generate these results, $q(0)$ was increased from its value at $\lambda=$ constant to $q(0)=1$ with a gradient in $\lambda$. The minimum value of $q$ increased along with $q(0)$ although not as rapidly. For the maximum $\beta_{p}$ on this curve, $q(0)=1$ and $q_{\min }=0.71$

The shear can be increased significantly if we relax the requirement that the $\lambda$-profile be monotonic, resulting in larger $\beta_{p}$, as also shown in Fig. 14. It is most conveniently parameterized by $q(0)-q_{\min }$, proportional to the average shear. The value of $q_{\min }$ is determined primarily by the maximum value of $\lambda$. As an example, the $\lambda$ and $q$ profiles for $\beta_{p}=0.124$ are shown in Fig. 15. The current profile corresponding to this case may require auxiliary current drive.

\section{References}

1. J. B. Taylor, "Relaxation of Toroidal Plasma and Generation of Reverse Magnetic Fields," Phys. Rev. Letters 33, 1139 (1974); "Relaxation and magnetic reconnection in plasmas," Rev. Mod. Physics 58, 741 (1986).

2. P. Gautier, R. Gruber, and F. Troyon, "Numerical study of the ideal-MHD stability limits in oblate spheromaks," Nucl. Fusion 21, 1399 (1981).

3. D. A. Kitson and P. K. Browning, "Partially Relaxed Magnetic Field Equilibria in a Gun-Injected Spheromak," Plasma Phys. and Controlled Fusion 32, 1265 (1990).

4. L. L. LoDestro and L. D. Pearlstein, "On the Grad-Shafranov equation as an eigenvalue problem, with implications for q solvers," Phys. Plasmas 1, 91 (1994).

5. T. R. Jarboe, F. J. Wysocki, J. C. Fernández, I. Henins, and G. J. Marklin, "Progress with Energy Confinement Time in the CTX Spheromak," Phys. Fluids B 2 , 1342 (1990).

6. E. B. Hooper, "Determination of spheromak flux conserver radius," Memorandum, September 5, 1996. 
7. M. N. Rosenbluth and M. N. Bussac, "MHD stability of spheromak," Nucl. Fusion 19, 489 (1979).

8. J. M. Finn, W. M. Manheimer, and E. Ott, "Spheromak tilting instability in cylindrical geometry," Phys. Fluids 24, 1336 (1981).

9. S. C. Jardin, M. S. Chance, R. L. Dewar, R. C. Grimm. D. A. Monticello, "Tilting and shifting modes in a spheromak," Nucl. Fusion 21, 1203 (1981).

10. Alan Turnbull, General Atomics, private communication January 10, 1997.

11. F. J. Wysocki, J. C. Fernández, I. Henins, T. R. Jarboe, and G. J. Marklin, "Evidence for a Pressure-Driven Instability in the CTX Spheromak," Phys. Rev. Letters 61, 2457 (1988).

12. Thomas R. Jarboe, "Review of spheromak research," Plasma Phys. Control. Fusion 36, 945 (1994).

13. M. Okabayashi and A. M. M. Todd, "A numerical study of MHD equilibrium and stability of the spheromak," Nucl. Fusion 20, 571 (1980).

14. R. M. Mayo and G. J. Marklin, "Numerical calculation of Mercier beta limits in spheromaks," Phys. Fluids 31, 1812 (1988).

15. G. Marklin, "MHD Stable High Beta Spheromak Equilibrium," Proc. US-Japan Workshop on Field-Reversed Configurations with Steady-State High-Temperature Fusion Plasmas and the 11th US-Japan Workshop on Compact Toroids, Nov. 1989, LANL report LA-1 1808-C, pp 181-284, May 1990.

16. S. C. Jardin, "Ideal Magnetohydrodynamic Stability of the Spheromak Configuration," Nucl. Fusion 22, 629 (1982).

17. S. O. Knox, et al., "Observations of Spheromak Equilibria Which Differ from the Minimum-Energy State and Have Internal Kink Distortions," Phys. Rev. Letters 56, 842 (1986). 


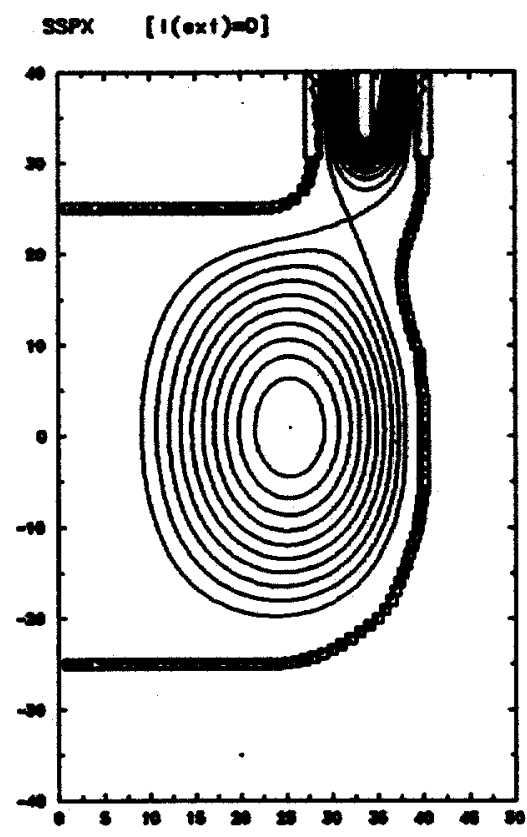

Fig. 1. The flux surface and flux conserver geometry; no current on open fieldlines. Note that the flux conserver is modeled by a large number of coils. The coaxial gun is modeled by the three solenoids at the top of the figure. (Dimensions in $\mathrm{cm}$.)

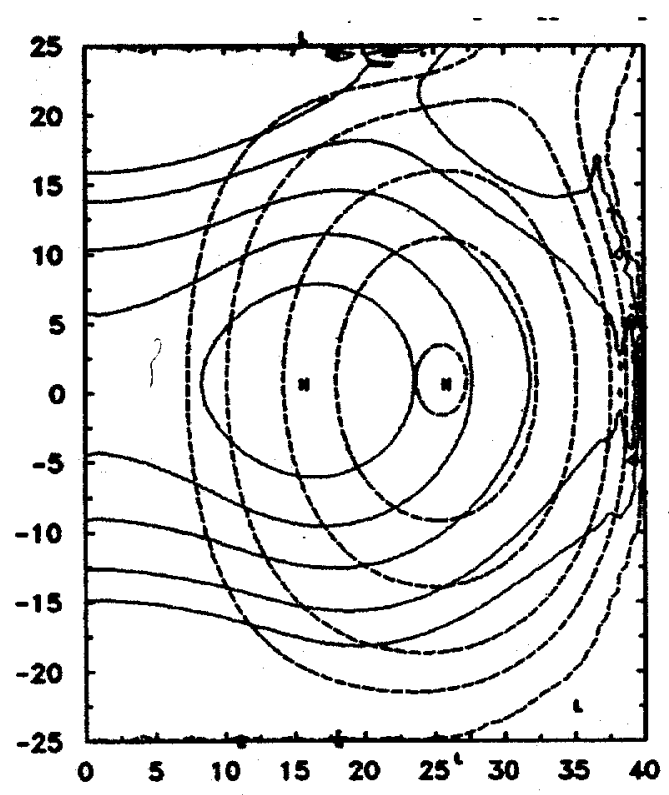

Fig. 2. $|\mathrm{B}|$ (solid lines) and flux surfaces (dashed). At $I_{\text {plasma }}=1$ $\mathrm{MA}$, the $\mathbb{B B}$ surfaces correspond to $2.0,1.7,1.4,1.1$, and 0.9 tesla. 

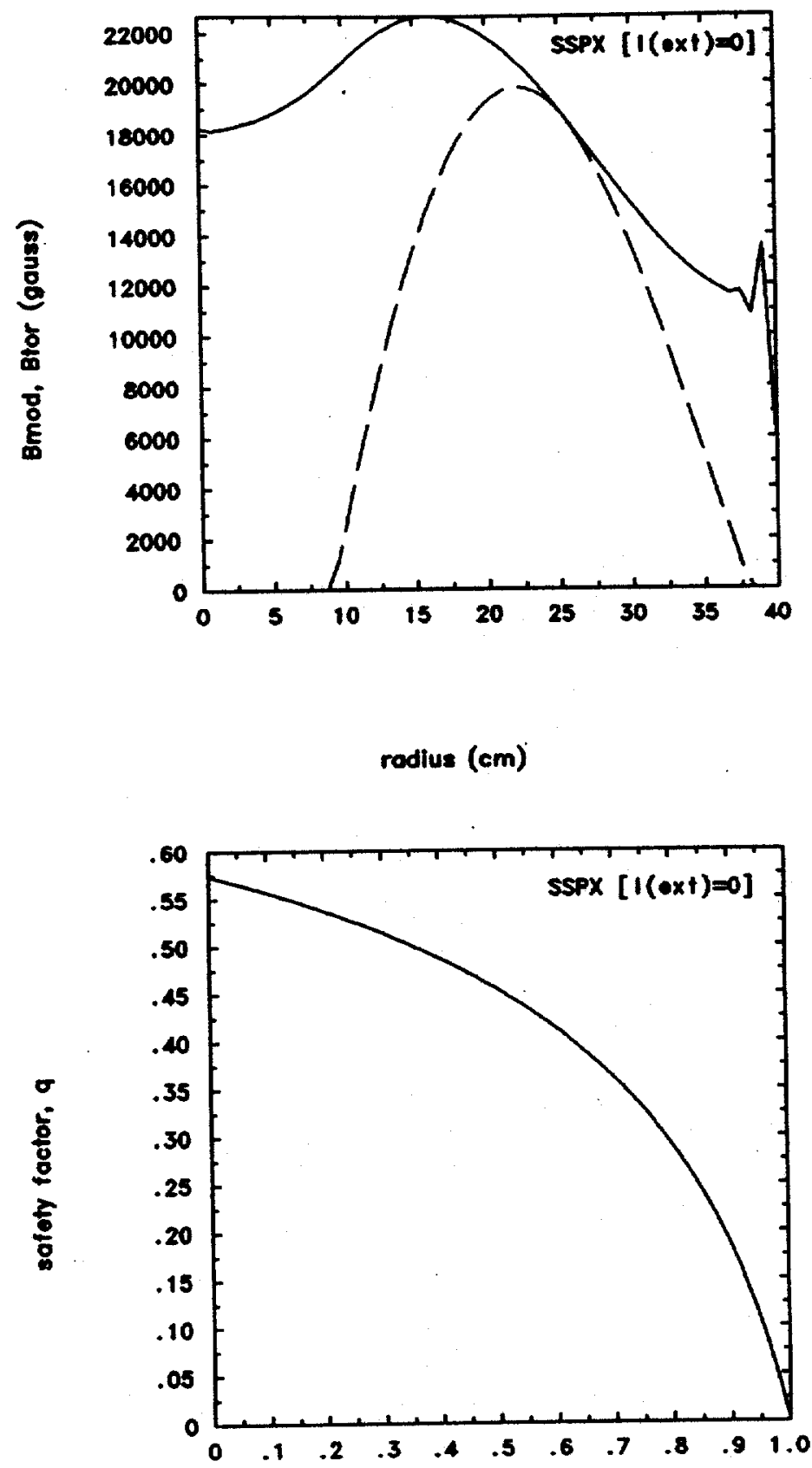

Fig. 3. $\mathbb{B} \mid$ (solid lines) and $B_{\text {toroidal }}$ (dashed) vs. radius on the spheromak midplane for toroidal plasma current $=1 \mathrm{MA}$. The structure in $|\mathrm{B}|$ at the edge is outside the separatrix and results from modeling the conducting wall by discrete coils.
Fig. 4. Safety factor vs magnetic flux. As there is no external toroidal magnetic field, $q=0$ at the plasma edge.

flux 

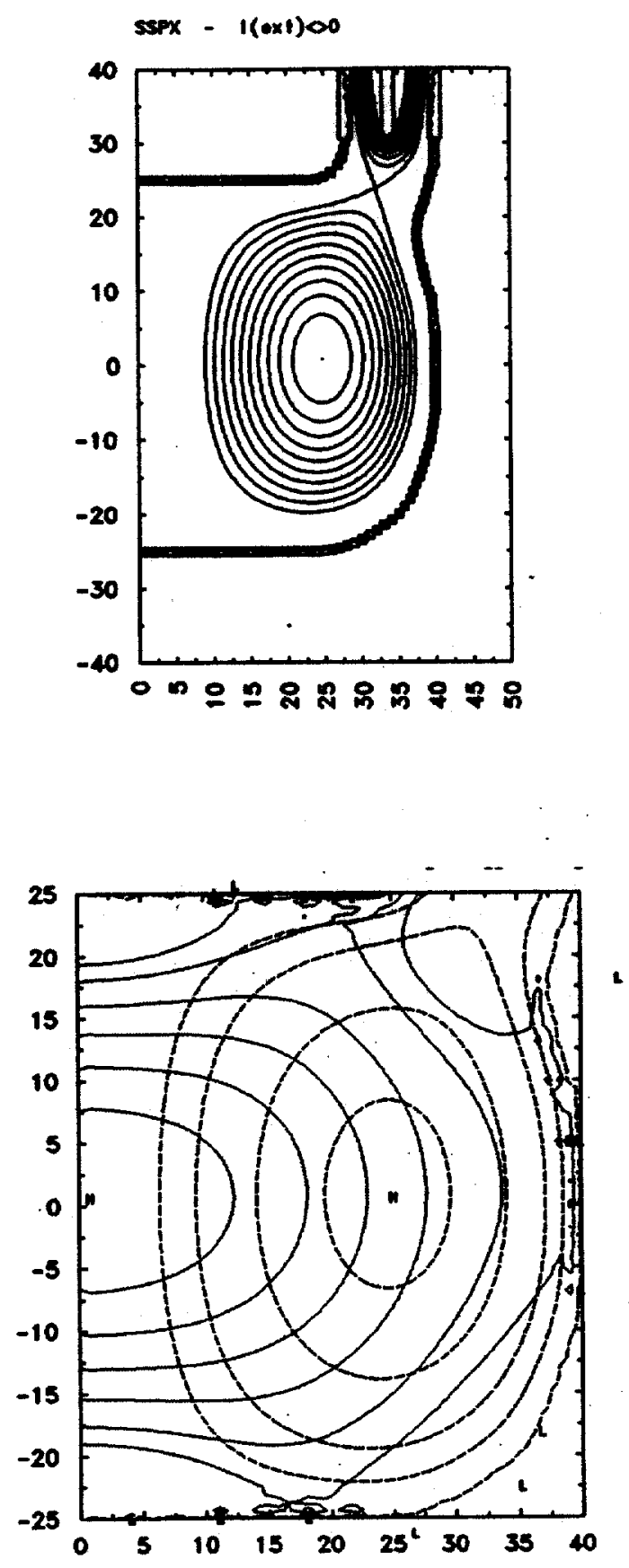

Fig. 5. Geometry for external current with $[I($ int $) / I($ ext $)=3.5]$; conditions otherwise as in Fig. 1

Fig. 6. $|\mathrm{B}|$ and flux surfaces corresponding to Fig. 5 . 

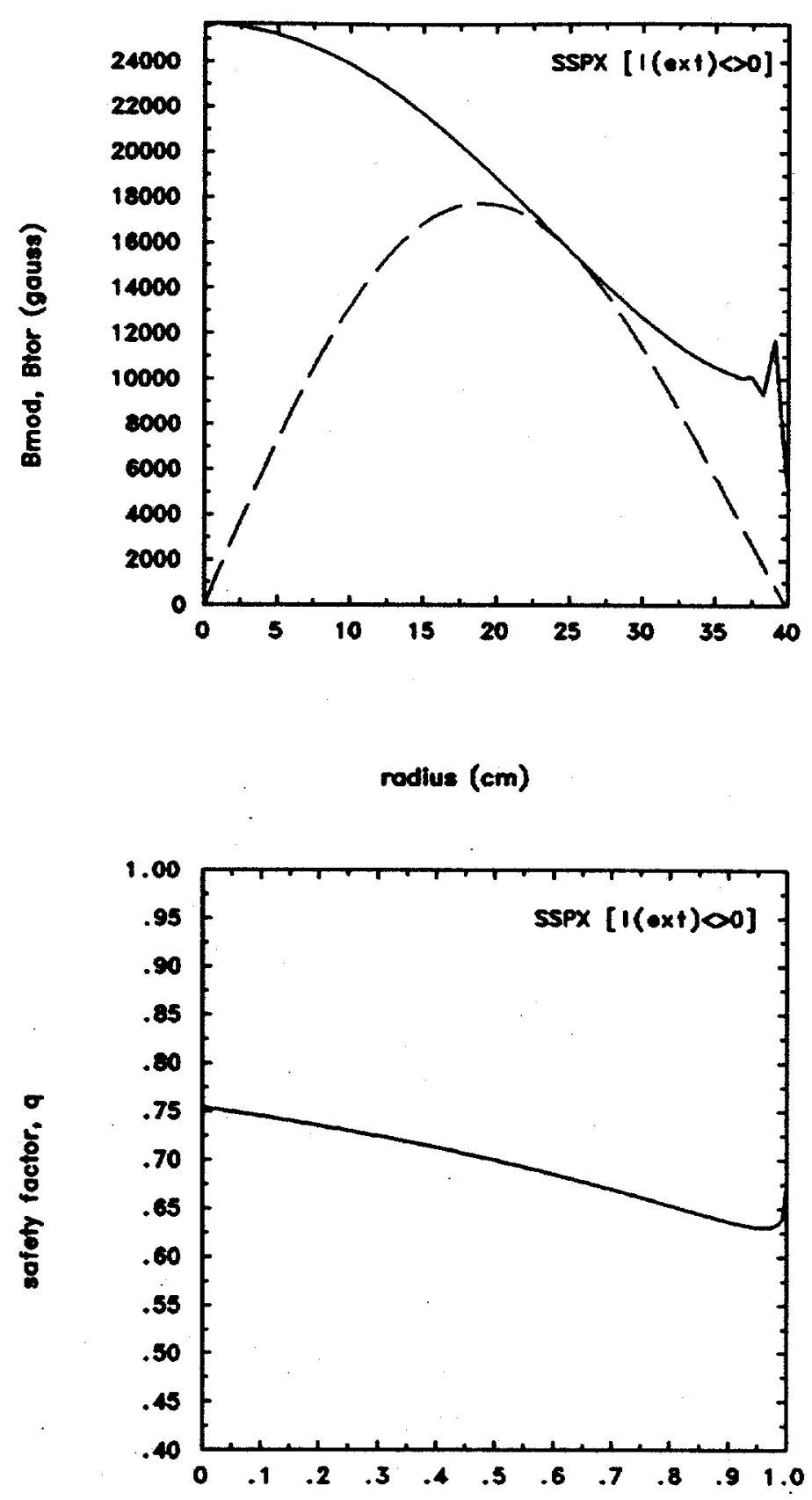

flux
Fig. 7. $|\mathrm{B}|$ and $\boldsymbol{B}_{\text {toroidal }}$ for the conditions of Fig. 5 . The toroidal plasma current within the separatrix = $1 \mathrm{MA}$.

Fig. 8. Safety factor for the conditions of Fig. 5. The divergence on the separatrix is not resolved at the grid spacing used. 


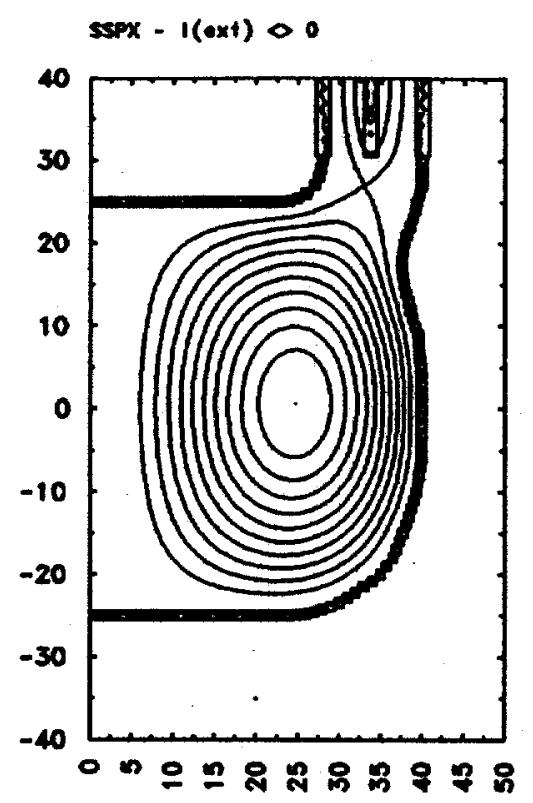

$\sigma$
$\frac{5}{8}$
$\frac{5}{8}$

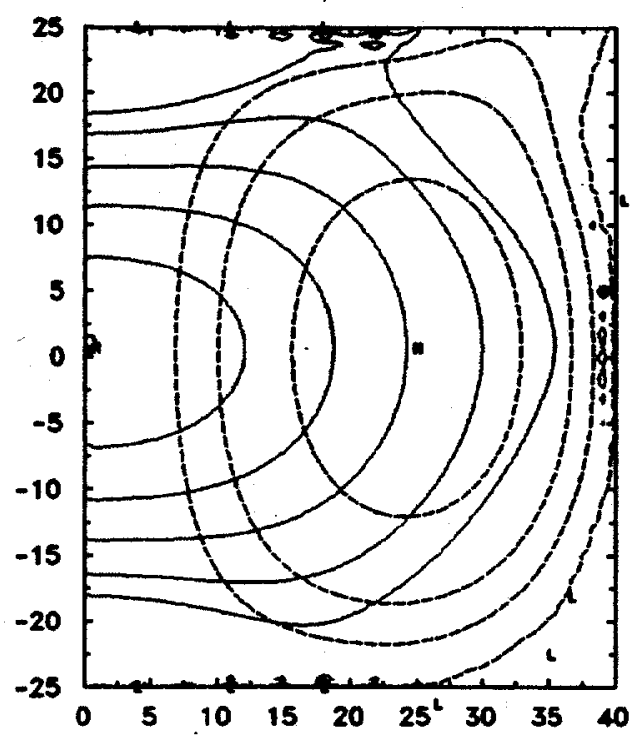

flux
Fig. 9. Geometry for external current with $[I($ int $) / I(e x t)=7.3$; conditions otherwise as in Fig. 1

Fig. 10. $|\mathbf{B}|$ and flux contours for the case of Fig. 9. 

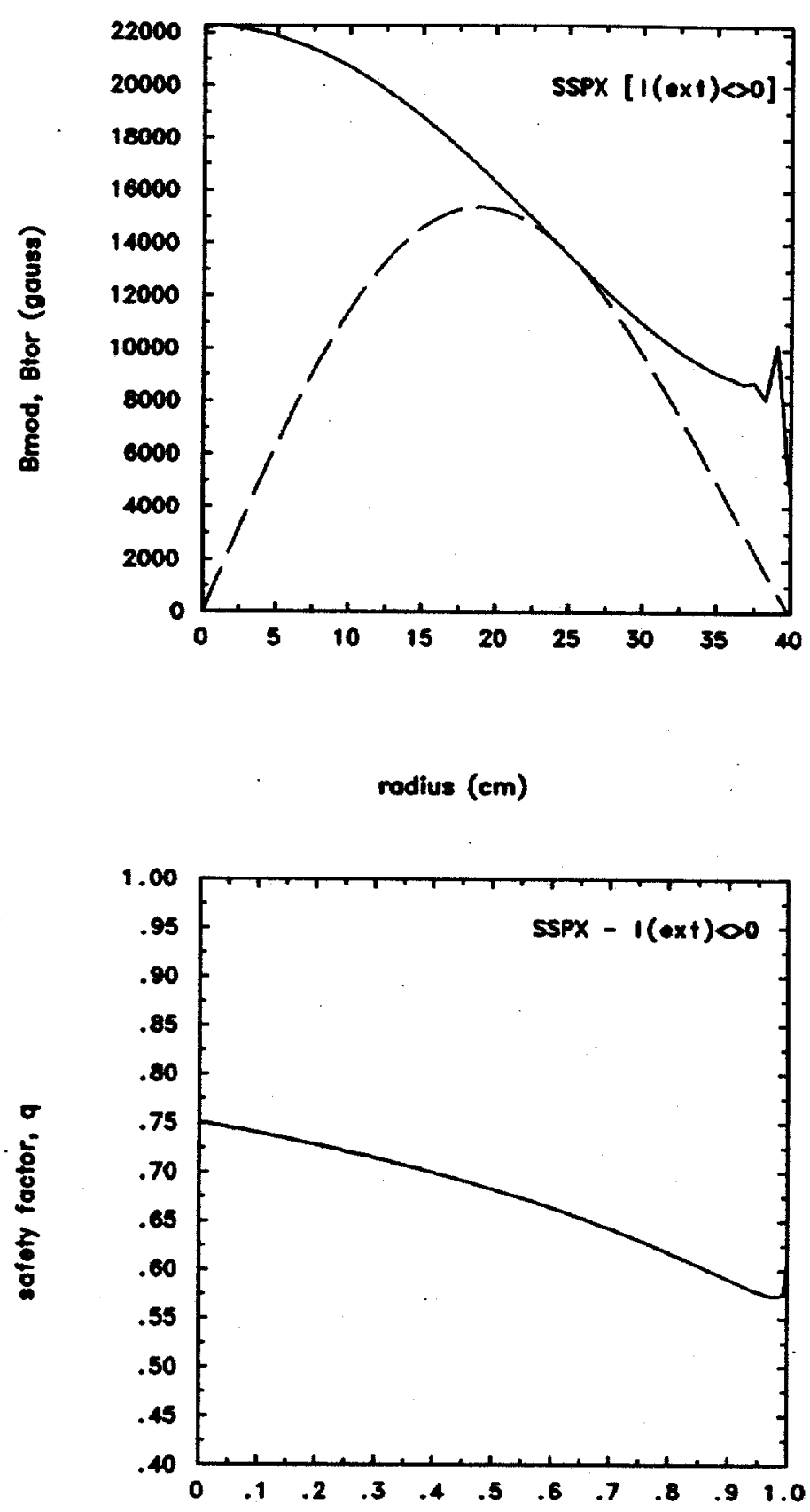

Fig. 11. $|\mathrm{B}|$ and $\mathrm{B}_{\text {toreidal }}$ on the plasma midplane for the case of Fig. 9. Plasma current (internal) $=1.0 \mathrm{MA}$.
Fig. 12. Safety factor for the case of Fig. 9. 
Fig. 13. Poloidal beta as a function of $q(0)$ (Fig. 1) with $I(e x t)=$ 0 so $q_{\min }=0$. The

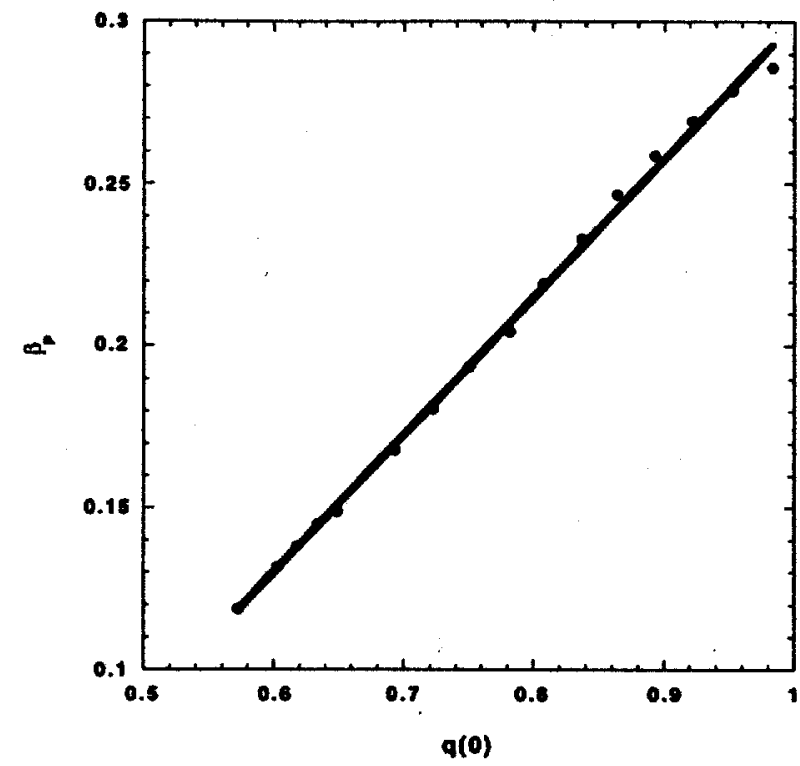
profile of $\lambda(\psi)$ was varied to increase the value of $q(0)$, as discussed in the text.

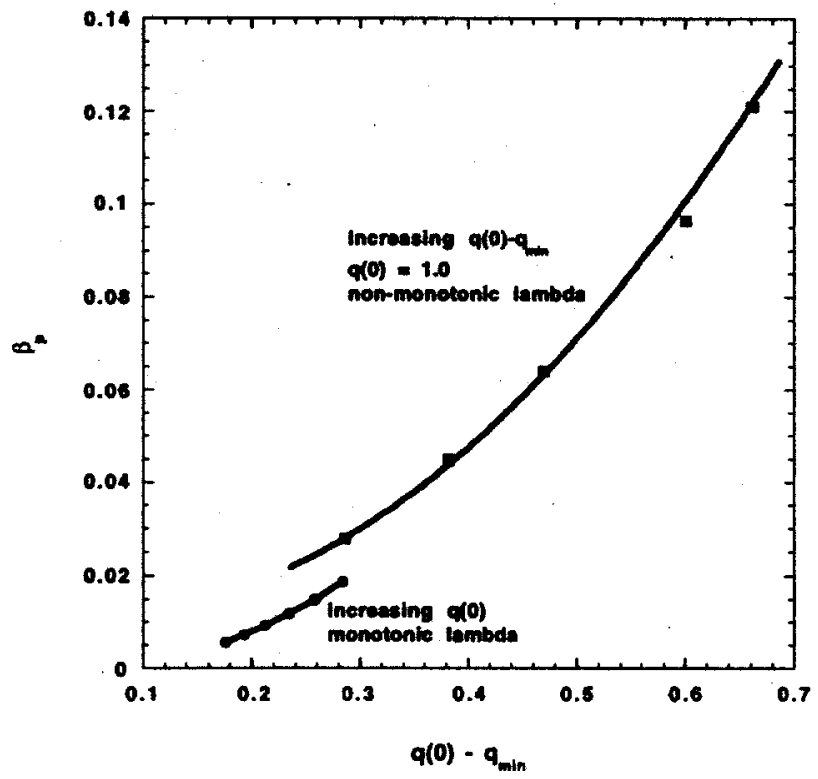

Fig. 14. Poloidal beta vs $q(0)-q_{\min }$ for external current (Fig. 9). The dots are for the case of the monotonic profile in $\lambda(\psi)$ as discussed in the text; maximum $\beta_{p}$ at $q(0)=1$. If $\lambda(\psi)$ is. not constrained to be monotonic, much larger betas are found. The maximum value of $\lambda$ is increased to lower $q_{\min }$ at $q(0)=1$. 


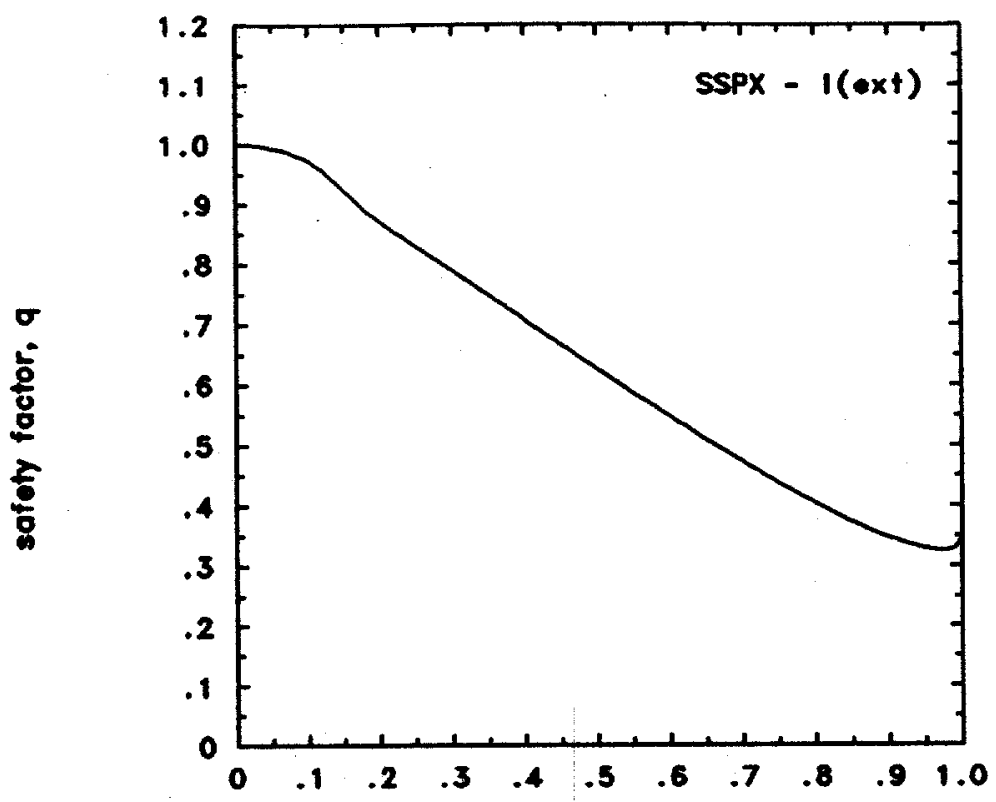

Fig. 15. $q(\psi)$ and $\lambda(\psi)$ for $\beta_{p}=0.124$. $I_{\text {ext }} / I_{\text {int }}=12.5$.

flux

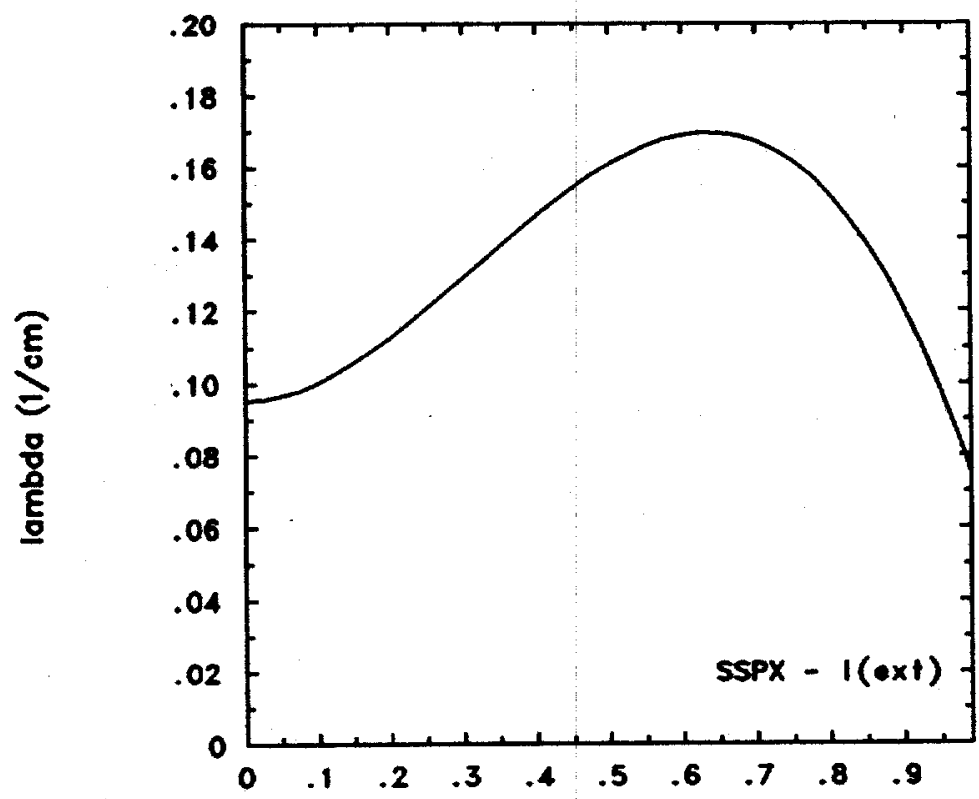

flux 


\section{Review of the MHD Stability of the Spheromak}

Although topologically spheromaks and tokamaks are basically the same, the MHD stability properties of the two configurations are quite different. It is instructive to compare the two with reference to the various classes of MHD modes, and we shall do so as appropriate, but in summary it can be said that the spheromak, lacking a strong toroidal field, is much more susceptible to ideal-MHD instability.

The literature specifically addressing stability of the spheromak is fairly extensive, including both analytic ${ }^{1-6}$ and numerical ${ }^{7-16}$ calculations. There is a variety of approaches and characterizations of equilibria, so that few close quantitative comparisons are possible, but results are in qualitative agreement, and understanding of the trends of parametric dependence is good. This review is intended in part as a guide to the literature (which occasionally seems to be in contradiction), as well as to the instabilities themselves. In what follows, $n$ and $m$ refer to toroidal and poloidal Fourier harmonics, respectively, of $\nabla \psi \cdot \xi$; pitch is defined as $q_{0} R_{0} / a$ (where $R_{0}$ is the average of the inner and outer plasma boundaries, as in a tokamak). Spheromak shapes are often described as oblate (flattened, compared to a sphere, about the symmetry axis) or prolate (elongated).

A summary picture of the stability properties of the spheromak can be given as follows.

The $n / m=1 / \pm 1$ current-driven modes (the tilt and horizontal shift) are always unstable. Sufficiently oblate spheromaks can be stabilized on the ideal-MHD timescale with an infinitely conducting wall. Feedback control (or other mechanism) will be necessary on the resistive-wall time-scale (as it is in elongated tokamaks for the $n=0$ vertical instability). The $2 / \pm 1$ modes probably fall into this same category, but one can design for stability without the wall to $n=0, n=3$, and higher perturbations.

In general, poloidal mode-number $m=1$ is dominant in spheromak instabilities. Toroidal mode-coupling appears to be weak, despite the often low aspect-ratios considered, due to the relatively low pressures and weak shaping. An exception to this is the case of high- $n$ instability of spheromaks with no central hole (separatrix on the symmetry axis), which is localized around the poles.

The periodic cylinder (i.e., large-aspect-ratio spheromak with a large, central hole), which is easy to analyse and for which it can be shown that stability is guaranteed if $m=1$ is stable at all $n$, can thus be expected to give a good qualitative picture of ideal toroidal computational results. $\operatorname{Jardin}^{9}$ provided useful analysis 
and comparisons here. The exceptions are the tilt, etc., which do not exist in the cylinder, and the $n / m=1 / 1$ resonant internal kink, which, though it has a cylindrical counterpart, behaves very differently in a torus.

Analogous to the external kinks in tokamaks, and well described by the cylindrical analysis, are nonresonant current-driven instabilities. In the tokamak, these are stable with a conducting wall on the plasma; with the wall at a distance, the $n / m$ ideal mode becomes stable as qedge increases and the associated resonant surface moves into the plasma from the vacuum; the instability (when the surface is outside the plasma) is driven by $J^{\prime}$ at the edge, so peaked current-profiles are better for stability. In the spheromak, the mode-rational surfaces successively enter the plasma on the magnetic axis as $q_{0}$ increases. Thus high $q_{0}$, and so hollow currentprofiles, promotes stability, and, since the relevant portion of the $J^{\prime}$ profile is near the axis rather than the edge, the wall is less effective, and there is a region of parameter space in which internal instability remains.

Beta is limited at lowest pressures by the just-mentioned current-driven modes. At intermediate $\beta$ 's, the Mercier limit becomes the sufficient (as well as necessary) condition for stability. The highly radially localized instabilities involved here are an exception to the $m=1$ rule mentioned above, but still appear to be at low-tomoderate $n$ and $m .^{8}$ (The Mercier criterion, discussed in Sec. 3.2, plays a much more interesting role in spheromaks than tokamaks.) As $\beta$ increases, $n>1, m=1$ resonant pressure-driven modes, localized between the magnetic axis and the moderational surface, set the limit. $\beta$ reaches its ultimate limit when $q_{0}$ reaches 1 , with the onset of the $1 / 1$ internal kink. Several authors ${ }^{8,9}$ have found ideal-MHD-stable volume-averaged $\beta_{\text {pol }}$ 's of the order of $30 \%$ in spheromaks with conducting walls at $r_{\text {wall }} / r_{\text {plasma }} \sim 1.2$; engineering $\beta$ 's, due to the low field at the walls, are significantly higher.

The current profile ( $\Leftrightarrow q$ profile) plays an important role throughout. We . have noted that broad or hollow current-profiles, with lower $B_{\mathrm{pol}}$ at the magnetic axis, are associated with high $q_{0}$. For spheromaks with $q_{\text {edge }}=0$, increasing pitch $q_{0} R_{0} / a$ promotes stability for all the modes of concern, until the $1 / 1$ resonant kink limit is reached: In the periodic cylinder, there is a critical pitch at a given wall distance above which the nonresonant current-driven modes are stable; the Mercier limit (proportional to shear), also goes up with the pitch; the tilt and shift modes can be stabilized with a more distant wall when the current is peaked at the edge, and furthermore, the drive decreases with aspect-ratio. Beta limits set by the resonant pressure-driven modes also increase with pitch. The cylinder, not subject 
to the toroidal $1 / 1$ current-driven modes, indicates an ultimate limit of $\beta_{\theta} \sim 70 \%$, independent of wall radius, set by the resonant $m=1$ modes.

High- $n$ perturbations, i.e., ballooning modes, have been found to be stable in spheromaks in all cases when the Mercier criterion is satisfied.

\subsection{Low-n Modes}

- CURRENT-DRIVEN. Stability limits at low $\beta$ are set by primarily nonresonant, global, parallel-current-driven modes dominated by the $m=1$ harmonic. With a conducting wall on the plasma, the modes are unstable in a region roughly characterized by $n q_{0}<1$ and $n a / R_{0}>1$.

In the first large numerical study of these modes Okabayashi et al. ${ }^{7}$ studied wall stabilization of an ideal-MHD oblate spheromak. The tilt was precluded by boundary conditions, and the vertical and horizontal shifts were stable with a distant wall. At large aspect-ratio $\left(R_{0} / a=6.7 ; q_{0}=.125\right.$; and $F^{\prime}=$ const. $)$ they found all modes $n<1 / q_{0}$ unstable, and a very few modes with $n>1 / q_{0}$ weakly unstable; the growth rate peaked at $n$ somewhat below $1 / q_{0}$. Modes with $n \leq 1 / q_{0}$ all had a similar, global structure. All modes could be wall stabilized, with $n \sim 1 / q_{0}$ requiring the closest wall.

Gautier et al., ${ }^{8}$ in the first ERATO study of the spheromak, considered equilibria with $q_{0}>.5$, so $n=1$ and 2 were the only possible $m=1$ modes with no resonant surface in the plasma. $n=1$, without the restrictive boundary conditions employed by Ref. 7, is dominated by toroidal effects and is discussed below. Growth rates of the free-boundary current-driven modes in Ref. 8 were observed to decrease monotonically with $n$ (Gautier remarks this differs from Ref. 7, but in fact Ref. 7 also found growth rates decreasing once $n>1 / q_{0}$, i.e., once the resonant surface lay inside the plasma). The $n=2$ mode was shown to be localized between the resonant surface and the plasma edge.

$\mathrm{Jardin}^{9}$ analysed the ideal stability of the infinite aspect-ratio spheromak in order to provide insight into the results of a wide survey of toroidal stability calculations using the PEST code. For the current-driven modes in the cylinder, using model $q$-profiles, he found a simple condition on the pitch (which increases as the current-profile flattens and hollows): $q_{0} R_{0} / a>2 / 3$ for stability to modes at all $n$ at zero pressure and $r_{\text {wall }}=r_{\text {plasma }}$. The stability boundary in $\left(n q_{0}, n a / R_{0}\right)$ space is fairly insensitive to wall position at low $\beta$, and weakly destabilized by $\beta$ if the wall is close; but as the wall moves out $\beta$ becomes very destabilizing. Jardin found the toroidal $n=3$ mode as obtained by PEST fairly well described in terms of its 
cylindrical-pinch counterpart (he did not look at toroidal $n>3$ ), whereas $n=1$ is dominated by toroidal effects. With the conducting wall at infinity, the $n=2$ mode is also driven by toroidal effects, and is unstable for equilibria of any pitch and oblateness; but as the wall approaches the plasma, $n=2$ becomes stable once $n q_{0} \gtrsim 1$, as in the cylinder. The toroidal results exhibit a minimum in instability with shape parameter, with best results (and best agreement with the cylinder) at moderate oblateness. Jardin also looked at the effects of lowering the magnetic shear at the magnetic axis; when $q \propto 1-r^{4}$, he found a region of internal instability around $n q_{0}=1$ cutting across the previously stable region $0<n a / R_{0}<1$. This eliminates the window of stability between the current- and pressure-driven modes in $\left(n a / R_{0}\right)-n q_{0}$ space.

The nonlinear development of these modes has been studied in the infiniteaspect-ratio limit by Park and Jardin. ${ }^{13}$ (Note that in this limit, due to symmetry in $\theta$ and $z$, no mode-coupling can occur.) A nonlinear kinetic-energy damping is added to the ideal-MHD simulation, so that new, stable, equilibrium states, if they exist, can be reached. With a conducting wall on the plasma, such helical states were found at fairly low saturation amplitudes, and without singular current layers (the linear mode being nonresonant); at $r_{\text {wall }} / r_{\text {plasma }}=1.3$, the saturation amplitude increased by $17 \%$.

These modes have been observed in CTX. In the course of resistive decay, the onset of the $n=2$ and 3 internal kinks correlates well with the loss respectively of the $2 / 1$ and $3 / 1$ surfaces at the axis. ${ }^{17}$ (The $n=1$ kink discussed below was also observed in CTX, during the helicity sustainment phase.) The instabilities are not disruptive to the equilibria, saturate at $\Delta B_{\mathrm{pol}} / B_{\mathrm{pol}} \sim 9 \%-10 \%$, and do not involve redistribution of the current toward a constant- $\lambda$ state. Knox et al. ${ }^{17}$ note the similarity with Ref. 13 and give some discussion of the lack of multiple reconnections and relaxation. It is possible, however, that the deduced spatially varying $\lambda$ were a consequence of using an axisymmetric fit procedure on what were in fät helical equilibria. As will be discussed below, there is evidence that with no resonant surfaces in the plasma, $\delta W_{\mathrm{JBT}}=\delta W_{\mathrm{MHD}}$, so that the saturation observed in CTX could be an indication that a succession $(n=0,1,2,3)$ of $\lambda=$ const. Taylor states was in fact achieved.

- PRESSURE-DRIVEN: modes driven primarily by $p^{\prime}$, with mode-rational surfaces in the plasma.

Beta-optimization was a main objective of the first ERATO study. ${ }^{8}$ The pressure 
profile was taken to be $p^{\prime} \propto \psi_{\text {axis }}-\psi ; F F^{\prime} \propto \psi(1+\alpha \psi)$, with $\alpha$ providing a knob on the width of the current profile; and $r_{\text {wall }} / r_{\text {plasma }}=1$. A family of moderately oblate $q_{\text {edge }}=0$ spheromaks was considered. A single shape-parameter, measuring hole size, or, equivalently, aspect ratio, was varied. For a given $\alpha$, the Mercier condition was checked on each rational surface. (For a given $n, m$ at the most stringent surface was generally low, and was not monotonic in $n$. Occasionally even here $m=1$ was most unstable.) The pressure was then increased relative to $F F^{\prime}$ (keeping the total current fixed) until some surface became marginal, yielding a volume-averaged total $\beta_{\max }\left(\alpha, R_{0} / a\right)$. Adjusting $\alpha$ to achieve $q_{0}=1$ was found to give the highest $\beta$ 's, after which $\beta_{\max }$ was observed to scale linearly with $R_{0} / a$, with $\beta_{\max } \sim 40 \%$ at $R_{0} / a=2.34$.

Gautier et al. also make an interesting observation regarding the numerical convergence properties of tokamak cf. spheromak stability calculations. Given an instability, ERATO's numerical error $\propto 1 / N^{2}$, where $N$ is the number of numerical elements; but because the calculation is not variational, the sign of the error can be in either direction. For tokamaks, it has always been found to be pessimistic, so that an instability is never missed with a coarse grid; for spheromaks, the error is of the opposite sign, and if no instability is found, it is not possible to predict whether one will turn up at higher resolution. Thus even the scoping surveys to identify the unstable regions of parameter space must be carried out at high resolution for spheromaks.

Pressure-driven modes were also studied by Jardin, ${ }^{9}$ who found the periodiccylinder model, mentioned above, useful here as well. In Ref. 9, the pressure is set to a constant $\alpha(0 \leq \alpha \leq 1)$ times a profile that is at each surface marginally stable to the Suydam condition, and $m=1$ stability boundaries (worst case in the cylinder) are found in $\left(n a / R_{0}\right)-n q_{0}$ space. With finite $\alpha$ and $r_{\text {wall }} / r_{\text {plasma }}=1$, there is instability in the region $n q_{0} \gtrsim 1$ and $n a / R_{0} \lesssim 1$, so that there is a window of stability. $\left(2 / 3<q_{0} R_{0} / a<1.1\right.$ for $\left.\alpha=1\right)$ between the current- and pressure-driven modes. Above $q_{0} R_{0} / a=1.1$, lower $\alpha$ is required for stability (the unstable region shrinks as the pressure is reduced below the Suydam limit). Nevertheless the maximum stable beta continues to increase with $q_{0} R_{0} / a$ : the increase of $\beta_{\text {suydam }}$ with $q_{0} R_{0} / a$ is more than enough to compensate for the decrease in $\alpha$. As $q_{0} R_{0} / a$ continues to increase and $\alpha$ to decrease, the competing effects cancel and the maximum stable $\beta_{\theta}$ approaches a limit $\sim 70 \%$. This limit appears to exist even as the conducting wall recedes, although higher $q_{0} R_{0} / a\left(\sim 2.6\right.$ at $r_{\text {wall }} / r_{\text {plasma }}=2$ and $\left.\alpha=.3\right)$ is required to reach it. The toroidal PEST results, with the Mercier criterion used similarly 
to set the pressure profiles, exhibit $p^{\prime}$-driven $n=2$ and 3 instability that seems to be well described by the cylindrical model. The beta limit continues to rise with $q_{0} R_{0} / a$ even while these modes set the limit. Well before the highest $\beta$-limits and saturation seen in the cylinder can be approached, however, the toroidal $1 / 1$ modes become dominant.

In the ERATO study, careful attention was given to the marginally stable modes (further discussed in Sec. 3.2), but with the optimization procedure described there was no reported evidence of the high-beta modes found by Jardin. Apparently in Ref. 8 , the critical surface considering all $n$ was one with $m>1$. It is possible that after the close analysis of their "standard case" (which had $q_{0} R_{0} / a \sim .8$ ), the bulk of the GATO survey work was carried out at $p=0$. But the fact that ERATO has shown a close connection between the Mercier criterion and the low- $n$ modes, together with the observation that the worst $m$ here is not always unity, raises a question about the toroidal beta-limits calculated in Ref. 9: Were worse modes, with $m>1$ and higher $n$, missed in Ref. 9 ? $n=3$ was the highest considered by Jardin, and even $n=3$ stability was not tested in the same portion of $\left(n a / R_{0}\right)-n q_{0}$ space as $n=2$ (only in the cylinder are these guaranteed to give the same result).

- TORUS ONLY: tilt, shift, and bending modes. The spheromak is subject to a variety of low- $n$ nearly rigid instabilities arising from the interaction of the plasma toroidal current-loop with the external vertical magnetic field necessary for equilibrium. These modes have no analogue in the cylinder. The $n / m=0 / 1$ mode is an axisymmetric vertical shift, well known in shaped tokamaks, driven by the gradients of the external field. There is a critical elongation in tokamaks, or prolateness in spheromaks, above which the mode is unstable. It is easily stabilized on the idealMHD time-scale with a nearby conducting wall, but due to the finite resistivity of the wall there remains instability on the wall $L / R$ time. The mode has then to be controlled with a feedback system on this slow time-scale. The design of control systems at reasonable power under reactor conditions is a critical issue for reactors, and there is thus considerable ongoing effort in this area in the design of ITER. At present there are well developed tools for vertical-stability analysis and control in realistic geometry, ${ }^{18,19}$ and present-day elongated tokamaks are routinely operated in this fashion. In the spheromak, a similar program must be carried out for the $n / m=1 / \pm 1$ global modes described next. (In spheromaks, since $n=1$ stability usually mandates an oblate design, the $n=0$ mode itself is often stable with the wall at infinity.) 
The tilt $([n / m]=[1 / 1]-[1 /-1])$ and shift $([n / m]=[1 / 1]+[1 /-1])$ instabilities are driven by the torque or force on the toroidal current loop by the external field in the event of a rotation (about a horizontal axis) or horizontal shift, respectively. The bending mode represents a nearly poloidally rigid $n=2$ toroidal deformation of the current loop (like a baseball seam).

Rosenbluth and Bussac, ${ }^{1}$ in their seminal study of the stability of near-spherical spheromaks in spherical conducting walls, found that with no central hole a combination of Taylor ${ }^{20,21}$ and ideal-MHD stability analyses predicts: with the wall on the plasma, prolate plasmas are unstable to $n=1$ perturbations and oblate are stable. With the wall at a distance, prolate plasmas require $r_{\text {wall }} / r_{\text {plasma }} \sim 1 / \epsilon^{1 / 5}$ for $n=0$ stability, where $\epsilon$ is the small parameter that measures the departure from a spherical plasma/vacuum separatrix shape; oblate plasmas are stable to $n=0$, and are stable to $n=1$ for $r_{\text {wall }} / r_{\text {plaema }}<1+.2|\epsilon| ; n=2$ requires $r_{\text {wall }} / r_{\text {plasma }}<1.18$ for stability at $\epsilon=0$.

Hammer ${ }^{2}$ subsequently studied ideal-MHD tilt-modes in spheromaks with nearly spherical separatrices and walls and with arbitrary pressure. He found that the Rosenbluth-Bussac condition on the conducting wall radius for stabilization of the tilt in oblate spheromaks continues to hold with arbitrary pressure; that optimizing the separatrix shape in this case yields a $50 \%$ improvement (increase in relative wall radius) and that prolate separatices can be wall stabilized by introducing $m=4$ deformations; that walls that fit closely at the poles are best; and that peaked current-profiles are more easily wall-stabilized.

A significant amount of computational work followed these studies, mapping out the parametric trends and optimizing low- $n$ stability.

Jardin et al. ${ }^{10}$ studied the ideal-MHD stability of the tilt and shift in a class of zero- $\beta$, moderately oblate to moderately prolate equilibria, varying the size of the flux hole (i.e., aspect ratio) and the conducting wall parameters. A model $q$-profile with $q_{0}=1$ was used. With no wall, they found the shift first becomes unstable in weakly prolate plasmas, with the growth rate increasing as oblateness increases; the tilt is unstable in the most prolate plasmas considered, and becomes stable as oblateness is increased. There is a gap in growth rate as a function of shape-parameter where the curves would cross, and the modes switch identity; and so there is instability at any value of shape parameter. (Note that Ref. 1 also found instability even for circular plasmas, without a wall.) With a conducting wall, it was found that $r_{\text {wall }} / r_{\text {plasma }}$ required for marginal stability increased with increasing aspect ratio. Examination of various conducting wall shapes (all topo- 
logical spheres) revealed that the shift is easier to stabilize with a wall than the tilt; that wall stabilization becomes significantly easier as aspect-ratio increases; that nearly horizontal walls across the poles are much more effective than a nearly vertical, cylindrical wall (in a subsequent letter, Jardin and Christensen ${ }^{11}$ showed that sets of passive figure-eight coils in these horizontal planes would suffice); and that with a wall everywhere equidistant from the plasma, even the most prolate plasma considered can be stabilized at $R_{0} / a=1.3$ with $r_{\text {wall }} / r_{\text {plasma }}=1.2 .^{*}$

Gautier et al. ${ }^{8}$ looked briefly at wall stabilization of low- $n$ current-driven modes. They emphasize that these modes, in contrast with internal modes, are destabilized by increasing $R_{0} / a$. We point out, however, that in their free-boundary calculations relaxed-state current profiles were used, so that $q_{0}$ dropped as aspect-ratio increased, whereas in their fixed-boundary calculations, as well as in Ref. 10's study of the tilt and shift, $q_{0}$ was fixed as aspect-ratio varied, and here the opposite conclusion was drawn; that is, increasing $R_{0} / a$ is stabilizing.

A more extensive application of ERATO to the study of the low- $n$ external modes was carried out by Pfersich et al. ${ }^{12}$ and concentrated on optimizing shape and aspect-ratio in order to minimize the number and virulence of the free-boundary modes with no conducting wall. Only zero-pressure, relaxed-state current profiles were considered. They found a large region of stability for the $n=3$ mode, but $n=2$ appears to be always be unstable (except perhaps for no flux-hole). They

* With the introduction of a central flux-hole comes some variation in usage of the terms prolate vs. oblate. A definition $\epsilon=.5 \kappa /\left(R_{0} / a\right)-1$, where $\kappa$ and $R_{0} / a$ are the elongation and aspect-ratio defined as for tokamaks, recovers Ref. 1's $\epsilon<,=,>0$ for oblate, spherical, and prolate spheromaks, respectively, at aspectratio unity, and measures the flattening of the object as a whole (as opposed to $\kappa$, which measures the shape of the plasma poloidal cross-section at a single toroidal angle). Using either $\epsilon$ or $.5 \kappa-1$ as a measure, all the shapes considered in Refs. 9 and 10 are oblate; but the most elongated geometries are nevertheless referred to there as "prolate." The authors seem to define prolate/oblate with respect to the marked, qualitative change as a function of shape-parameter, at the point where the tilt and shift growth-rates cross. It is not known whether as the aspect-ratio decreases exactly to unity (and the central hole disappears - sudden big difference in $q_{0}$ ), the crossing moves toward $\epsilon=0$ and the Rosenbluth-Bussac result (internal tilt unstable if $\epsilon>0$ ) is smoothly recovered. This point is discussed in Ref. 12, where caution is advised; results approaching the limit suggest that it is singular. 
conclude that a slightly oblate shape and aspect-ratio near unity is best. Here again there seems to be confusion about increased stability at lower aspect-ratio. In their discussion, Pfersich et al. remark, on the basis of the non-monotonicity of their $n=1$ growth rates $v s$. oblateness in conjunction with monotonic $q_{0} v s$. oblateness, that the effect cannot be attributed just to increasing $q_{0}$ with decreasing $R_{0} / a$ (a consequence of relaxed-sate profiles). But we point out that if $\gamma$ decreases with $q_{0}$ more weakly for more prolate plasmas, then it is possible that the difference in the structure of their $n=1$ results compared with Ref. 10's (whose Fig. 6 indicates a minimum in growth rate vs. oblateness) is entirely due to varying $q_{0}$.

A fixed current down the symmetry axis helps stabilize the $n=1$ modes. Jardin et al., ${ }^{10}$ using the PEST code, report that with $q_{0}$ fixed at 1 , the tilt and shift eigenfunctions change continuously with increasing $I_{\text {axis }}$ to a pure $1 / 1$ external kink, which becomes stable when $q_{\text {edge }}=1$, at which point the configuration is a low-aspect-ratio tokamak. Yamada et al., ${ }^{22}$ in a recent study, find more generally that the unstable $1 / \pm 1$ modes change into $n=1, n q_{\text {edge }}=m$ external modes. They report good agreement between various rigid tilt/shift calculations and experimental data on $n=1$ MHD stability in the TS-3 low-aspect-ratio tokamak. At elongation

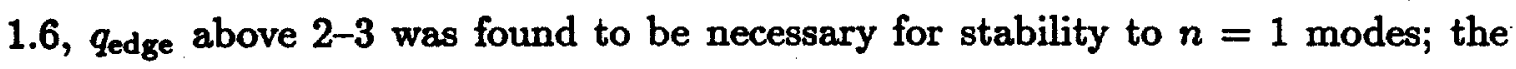
threshold $I_{\text {axis }}$ as a fraction of (fixed) plasma current increased strongly with aspect ratio, with .2 the value at $R_{0} / a=1.1$.

Finally, we remark on the possibility of rotational stabilization of the tilt and shift. As is well known from experience with tokamaks, in the presence of rotation, resistive walls can sometimes behave as if infinitely conducting. ${ }^{18}$ An initial study exploring these effects on the tilt has been carried out by Zweibel and Pomphrey. ${ }^{23}$

- $q_{0}>1$ KINK (pressure- and current-driven).

Within the large-aspect-ratio cylindrical-plasma approximation, an $m=1$ rigid displacement reduces the perturbed magnetic energy in the plasma to zero, its minimum value. The perturbed pressure contribution is destabilizing. If there is a $q=1 / n$ resónant surface in the plasma, a zero-energy jump in $\xi_{r}$ is possible, and thus the internal kink - a rigid displacement inside the resonant surface and zero displacement outside-will be marginally stable at $\beta=0$.

In the torus with zero pressure, stability depends on the sign of the higherorder corrections in inverse aspect-ratio, and thus a clear understanding of this mode in tokamaks proved to be a challenge. Bussac et al. ${ }^{24}$ showed that at large aspect-ratio only $m=2$ coupling need be considered, and they obtained $\delta W \propto(1-$ 
$\left.1 / n^{2}\right) \delta W_{\text {cyl }}+\delta W_{\text {toroidal }} / n^{2}$, where for the cases they considered $\delta W_{\text {toroidal }}$ was found to be numerically smaller than $\delta W_{\text {cyl }}$. Thus it is clear that $n>1, m=1$ modes are essentially the same as in the cylinder, but $n=1$ modes are fundamentally different. (We note that the Bussac et al. paper did not settle the issue. A clearer calculation, substantiating the results and elucidating the four parametric dependences, was later presented by Zakharov. ${ }^{25}$ ) Assuming $1-q_{0} \ll 1$, and $r_{\text {wall }} / r_{\text {plasma }}=1$, Bussac et al. found critical $\beta_{\text {pol }}$ as a function of $r_{1 / 1} / a$ : With $q / q_{0}-1 \propto r^{2}, \beta_{\text {crit }} \sim .3$ at $q_{0}=1$ and decreases as the resonant surface moves out until eventually $q_{\text {edge }}<2$, after which wall effects enter and $\beta$ is stabilizing. With weaker shear at the axis $\left(q / q_{0}-1 \propto r^{4}\right), \beta_{\text {crit }} \sim .08$ when $q_{0}=1$ and increases as $r_{1 / 1} / a$ increases.

$\mathrm{Jardin}^{9}$ applied these toroidal calculations to spheromak geometry with $q / q_{0}-$ $1 \propto-r^{2}$. He found that $\beta$ above $\beta_{\text {crit }}$ is required for stability, with $\beta_{\text {crit }} \sim .3$ for $q_{0}=1$ and increasing as a function of $r_{1 / 1} / a$.

Gautier et al. ${ }^{8}$ found a threshold $q_{0} \sim 1.07$ for the current-driven internal kink in a moderately oblate spheromak, and showed that the growth-rates are indeed reduced with the addition of pressure.

\subsection{High- $n$ Modes; Mercier Condition}

Employing a Taylor analysis, Rosenbluth and Bussac ${ }^{1}$ found that currentdriven high-n modes in a spheromak with no central hole are always unstable; specifically, they showed that $1-r_{\text {plasma }} / r_{\text {wall }}<.75 / n$ is required for stability in the large- $m$ limit. The high- $n$ modes are localized to the surface and to the vicinity of the poles. At large aspect-ratio (i.e., Taylor states in the limit of a large central hole), a similar result was obtained. The latter result can be compared with Jardin's previously mentioned condition for ideal current-driven modes with $r_{\text {wall }}=r_{\text {plasma }}$ in the cylinder, which was determined by an expansion of the Euler-equation near the magnetic axis. Jardin used a model profile $q=q_{0}\left(1-r^{2} / a^{2}\right)$; with the Taylorstate $q=q_{0}\left(1-(2.4 r / a)^{2} / 8+\cdots\right)$, the condition is $q_{0} R_{0} / a>.67 * 2.4 / \sqrt{8}=.57$ for ideal stability. The large-aspect-ratio Rosenbluth-Bussac Taylor analysis, on the other hand, predicts marginal stability at $q_{0} R_{0} / a=.83$, indicating that a significant portion of the ideal stability window in $\left(n q_{0}, n a / R_{0}\right)$ space is unstable to relaxation processes. Ref. 1 suggests that smoothly vanishing edge-current profiles or FLR could eliminate these instabilities without having much impact on the low- $n$ results. Turning to the interchange, Rosenbluth and Bussac find very low $\beta$-limits due to the particularly low shear in spheromaks with no central hole $\left(B_{\text {tor }} / R\right.$ and so $q$ is then finite at the edge); they estimate $\beta$ 's $\sim 2 \%-3 \%$ for oblate spheromaks 
with a central hole or modified current profiles.

In their numerical study, Okabayashi et al. ${ }^{7}$ dealt at high-n only with the pressure-driven modes. They found equilibria self-consistent with pressures everywhere at the Mercier limit, and observe that: pressure profiles tend to be flat, with $p^{\prime}$ supported at the edge where the shear is high; on-axis $\beta_{0}$ at first increases with the elliptical separatrix elongation, $\kappa_{s}$, has a maximum at $\kappa_{s} \sim .5$ (the surfaces near the magnetic axis at this $\kappa_{s}$ are nearly circular), and then decreases; $\beta_{0}$ increases sharply as the current profile $\left(F F^{\prime}\right)$ flattens and $q_{0}$ rises, while for fixed $F F^{\prime}$ shape, $\beta_{0}$ increases rapidly with aspect-ratio at low aspect-ratio and then saturates (as $q_{0}$ declines). Optimization yielded $\beta_{0} \sim 2 \%$. With a hollow current profile, a higher $q_{0}=.75$ was obtained; this yielded $\beta_{0} \sim 14.6 \%$ at $R_{0} / a=2$.

Gautier et al. ${ }^{8}$ also give an interesting discussion of the Mercier criterion in spheromaks, pointing out the contrasts with tokamaks. In the latter, the condition is usually most stringent at the magnetic axis. At large aspect-ratio and with circular surfaces, the condition reduces to

$$
\frac{B_{\text {tor }}^{2}}{8 \mu_{0} r}\left(\frac{r q^{\prime}}{q}\right)^{2}>-p^{\prime}\left(1-q^{2}\right),
$$

so that for normal pressure profiles it is satisfied at any shear if $q_{0}>1$. (More generally, the stabilizing right-hand side has to overcome destabilizing aspect-ratio and quadratic $p^{\prime}$ contributions, and is least negative on axis.) In a spheromak, the right-hand side is positive and shear stabilization is required. With normal current profiles and $p$ parabolic in $\psi$, the axis still sets the beta limit; however, as $q_{0} \rightarrow 1$ and the beta limit increases (and the current-profile hollows), the least stable point moves out to normalized flux-surface $\sim .85$.

Gautier finds that the resonant surface that sets the Mercier beta-limit is at low to moderate mode-number, and furthermore that this limit coincides with fixedboundary results from ERATO; in other words, the Mercier condition here is not just necessary, but sufficient for internal low- $n$ ideal stability, and the radially localized perturbations that set the limiting beta [in the stable window in $\left(n q_{0}, n a / R_{0}\right)$ space] are in fact low- $n$ modes.

Above the Mercier limit, ERATO gives growth rates that are extremely small until $\beta$ reaches nearly three times the limiting $\beta$; various non-ideal effects would enter here, and perhaps play a role in explaining experimental results indicating stability above the Mercier limit. Volume-averaged $\beta$ at the Mercier limit, fixing $q_{0}=1$, was found to increase linearly with hole size, and reached $40 \%$ at an-aspectratio of 2.34 . 
In the limit $n \rightarrow \infty$, satisfying the ballooning stability condition (necessary \& sufficient at $n \rightarrow \infty$ ) guarantees satisfying the Mercier condition (for general $n$, necessary but not sufficient). However, Refs. 7-9 find in all cases that the ballooning modes are stable when the Mercier condition is satisfied. Okabayashi observes that small edge $B_{\text {tor }}$ results in weak coupling to toroidal curvature where the pressure gradient is steep. Greene and Chance, ${ }^{28}$ invoked to explain these results in Refs. 8 and 9, have shown that when the magnetic shear is negative, then Mercier stability coincides with ballooning stability up to to very high values of shear. This calculation was carried out at $q=1.5$, however, and should be reexamined before applying it to $q<1$.

\subsection{Resistive Modes}

Taylor ${ }^{20,21}$ conjectured that tearing-mode stability is naturally assessed within the framework of his relaxed-state minimization procedure. That is, if an examination of the non-axisymmetric equilibrium states yields only higher eigenvalues $\lambda$, then the axisymmetric configuration is stable to all resistive and ideal-MHD modes. If true, this conjecture would clearly be very useful, and it has received wide attention. While no conclusive statement can yet be made, it seems to be born out by or at least consistent with results in practice. We give three examples of its application to spheromaks, where the idea has played a role in most of what is known about tearing.

Finn et $a l .{ }^{3}$ have given a proof that while in general the Taylor $\delta W_{\mathrm{JBT}}<\delta W_{\mathrm{MHD}}$, if there is no mode-rational surface in the plasma, then $\delta W_{\text {JBT }}=\delta W_{\text {MHD. The sign }}$ of $\delta W_{\mathrm{JBT}}$ is determined by the sign of $\lambda-\lambda_{\mathrm{axisym}}$. They then examine a force-free spheromak bounded by a conducting, capped cylindrical wall with no central hole, and find $n=1$ instability above a critical $L / R=1.67$, where $R$ is the radius of the cylinder and $L$ its length in $z$, for both Taylor and ideal-MHD analyses; above $L / R=1.793$, where the $q=1$ mode-rational surface first enters the plasma at the magnetic axis, the ideal case goes through a second marginally stable point, whereas the Taylor approach predicts continued instability. (Note that Ref. 3 uses the symbol $m$ for toroidal mode number, and that many poloidal harmonics are present, coupled by the cylindrical-can boundary conditions.)

Similarly, in their application of Taylor's approach to spheromaks, Rosenbluth and Bussac ${ }^{1}$ found that in the limit of large aspect-ratio (large central hole), the ideal-MHD and.Taylor results are the same for $m=1$ modes with long wavelengths (in $z$ ), where no singular surfaces appear. At shorter wavelengths, on the other hand, 
the ideal-MHD $m=1$ perturbations are localized between the singular surface and the edge, and become stable, while here the Taylor-state analysis indicates instability (although at least the $R_{0} / a=1$ Taylor-analysis large- $n$ modes were also localized to the surface). Rosenbluth and Bussac were thus led to speculate that the $n \geq 2$ current-driven modes with no hole are ideal MHD for small $n$ and tearing for large $n$.

Heidbrink et al. ${ }^{14}$ carry out a direct test calculation. In their study of tearing in a spheromak, which also employed the periodic-cylindrical-plasma model, they report excellent agreement between Taylor's prescription and a $\Delta^{\prime}$ calculation of the resistive modes in a relaxed-state equilibrium. Heidbrink also mentions that Newcomb's comparison theorem for ideal-MHD modes in the cylinder (if stable to $m=1$ for all $n$, then stable to all $m>1$ ) was observed to hold for tearing in all equilibria they examined.

The precise agreement with $\Delta^{\prime}$ found by Heidbrink is perhaps surprising. In a minimization formalism for force-free equilibria developed by Jensen et al. ${ }^{29}$ which allowed for a variable number of constraints, conditions for stability to tearing perturbations were located between ideal MHD and Taylor's condition. Applying these tearing-principle constraints in tokamak ordering, standard tearing eigenvalue equations were recovered. Taylor's principle, much less constrained than this, might be expected to find $\delta W_{\mathrm{JBT}}<0$ and so predict continued instability at the point marginally stable to tearing. [However, since the modes allowing access to this lowest-energy state are presumably on the slow resistive diffusion time-scale ( $q$ not conserved on any surface), which is the same as the transport time-scale, even if $\delta W_{\mathrm{JBT}}$ is significantly lower than $\delta W_{\text {tearing }}$ it may be that the latter is the more appropriate functional to minimize.] On the other hand, a proof ( $c f$. Finn's ${ }^{3}$ for the nonresonant case) may yet be found that when there are mode-rational surfaces in the plasma, then $\delta W_{\mathrm{JBT}}=\delta W_{\text {tearing. }}$.

The resistive interchange in spheromaks is an even more serious concern. Since the configuration is not minimum- $B$ and positive shear is here (as opposed to Eq. 3.1) required to stabilize normal pressure profiles, the unfavorable curvature is predicted to drive the mode unstable at any $\beta$. Non-ideal effects are then required for stability. There have been a number of works which enlarge the scope of the physics treated with Taylor's approach, including finite pressure-gradients, ${ }^{30-34}$ multiple species and equilibrium flow. ${ }^{35-39}$ (In fact, Woltjer's original papers ${ }^{40}$ on minimization principles for stable equilibria include both pressure and flows.) 
These are discussed briefly by Taylor ${ }^{21}$ and the more recent works are reviewed by Steinhauer. ${ }^{41}$ One might consider adding, say, (stabilizing) FLR, in an attempt to treat the resistive-g in a manner similar to tearing. However, while the constrained minimizations associated with such extended formulations offer a relatively easy method for calculating the stability properties when more realistic physics is included, the minimization principles themselves lack the convincing support from experiment as well as the recent support from theory ${ }^{42}$ available for the minimum$E /$ conserved- $K$ principle.

In summary, pressureless spheromak equilibria have been found with aspectratios and $q$-profiles of interest to be stable to tearing modes, but the major parametric studies carried out for ideal modes remain to be done. If the circular cylindrical plasma remains a good rough guide, as it has been shown to be for ideal modes (apart from the exceptions noted above), and as is generally the case for tearing modes in tokamaks, ${ }^{43}$ the results of Ref. 14 indicate that: $m=1$ is the most unstable mode; there is a critical pitch above which the $m=1$ is unstable at some (moderate) $n$; broad current-profiles are more favorable (as they are for the nonresonant current-driven ideal modes); and wall stabilization is ineffective (unless it is extremely close and the rational surface is near the plasma edge). Nonideal effects are required to stabilize the resistive interchange in the spheromak, and the collisionless trapped-ion mode is similarly a concern. 


\subsection{MHD Issues Remaining to be Addressed}

There remain a number of MHD issues requiring attention in order to be able to assess the attractiveness of the spheromak as a reactor, as well as questions to be addressed for SSPX:

- Formalism for the control of the $n=1 \mathrm{tilt} / \mathrm{shift}$ and the $n=2$ bending mode must be developed and implemented in a free-boundary equilibrium code such as CORSICA. In the near term, estimates of the power requirements can be obtained using $\gamma \tau_{L / R}=W_{\mathrm{MHD}}^{(2)} /\left(W_{\text {wall }}^{(2)}-W_{\mathrm{MHD}}^{(2)}\right)$, as has been done for vertical control studies; $\tau_{L / R}$ and the energies are to be obtained from, e.g., GATO. T. K. Fowler has suggested that the formalism developed for the whirl instability of mechanical rotors could serve as a useful simple model for studying the control of the resistive-wall mode with rotation.

- The stability and variation with parameters of a spheromak plasma with current on the open field-lines and limited by a surface with an X-point $\left(\Rightarrow q_{\text {edge }} \rightarrow \infty\right.$ ) remain to be explored.

- The low overall magnetic shear and the consequences for beta-limits with infinite $q_{\text {edge }}$ are a concern. Two variations of the SSPX design might address this problem: One could add at the outermost (with respect to the magnetic axis) open fluxsurfaces another current layer, about equal in magnitude and of opposite sign to the open-field-line current in the present designs, thereby converting the configuration to an edge-sustained RFP; or, one could drive the open-field-line current only on the outboard side, so that $B_{\text {tor }}$ on the inboard open surfaces remains zero. Either of these options results in low or zero $q_{\text {os. }}$. The equilibria could be scoped out fairly quickly with TEQ.

- The current-driven $n=1$. modes in particular need study with a current $I_{\text {axis }}$ on the open field-lines. Sufficient $I_{\text {axis }}$ should be unstable (as in a $z$-pinch), and this is a candidate mechanism for supplying helicity to the core plasma. On the other hand, there is the danger of these modes coupling to and driving unstable the $n / m=1 / \pm 1$ tilt/shift. Line-tied and insulating boundary conditions should be investigated, with lines terminating at various places (at the bottom of the vessel or all the way around back to the gun). It would be good to include rotation, possibly sheared (which would promote edge-localized effects), in the calculation. Significant rotation was observed in CTX, and would be driven (toroidal and poloidal) in the equilibria with reversed current on the outermost open surfaces mentioned above.

- Resistive modes in spheromaks, although studied in the large-aspect-ratio limit, 
have not yet been assessed in toroidal geometry. There is some discussion of what might be done in this regard in the section above on resistive interchange modes.

- In their numerical study of pressure-driven modes, Gautier et al. ${ }^{8}$ observed very low growth-rates for $\beta$ 's up to $\sim 3 \beta_{\text {Merc }}$; and in Ref. 44 , evidence is presented for pressure-driven instability whose onset was observed to occur at $\beta$ 's $\sim 20$ $40 \beta_{\text {Merc }}$. An effective $\beta$-limit significantly exceeding $\beta_{\text {Merc }}$ has important, favorable implications for reactors. These results thus suggest a theoretical study in which first, the Gautier et al. results are verified at the higher numerical resolutions now possible (GATO has the required capabilities); second, the suppression of MHD growth-rates is studied and the range of the effect understood as a function of mode-number and equilibrium parameters; and finally, the appropriate small nonideal effects that come into play and determine the actual stability thresholds in these regions are identified.

- The calculation of Greene and Chance, ${ }^{28}$ showing that if the magnetic shear is negative, then the Mercier limit is sufficient as well as necessary for local stability (up to large values of $\left|q^{\prime}\right|$ ), was carried out numerically by perturbing about a $q>1$ equilibrium. The calculation should be reexamined for $q<1$ in order to be able to apply the result with confidence to spheromaks. There is also perhaps an interesting second-stability calculation on the $1 / 1$ kink: The finding of Jardin ${ }^{9}$ that $\beta$ stabilizes the mode, but not until $\beta$ is high, violating the Mercier limit, in combination with the above-mentioned apparent possibility of effective MHD stability above the Mercier limit, could provide a route to quite high- $\beta$ spheromaks.

- (long term.) The quantitative study of spheromaks on the transport time-scale is a daunting project. An efficient approach, which exploits the multiple timescales of the problem, would proceed by coupling 1D current-transport (as well as $p$ and $n$ if desired) and circuit-control equations to a 3D equilibrium code (e.g., the PPPL PIES code): that is, by applying the Grad-Hogan approach to a spheromak. A 3D, rather than an axisymmetric 2D, equilibrium calculation is called for because the instabilities that must be controlled are $n=1$ and $n=2$. It has been shown in tokamak applications ${ }^{\mathbf{4 5}}$ that nonlinear effects can enter at very low amplitudes of the nonaxisymmetric perturbations, and that tearing modes can have a significant impact, so that the power requirements obtained from the linearized CORSICA calculation mentioned above could be significantly underestimated. Apart from efficiency, accuracy in resolving and confidence in the magnetic-field structure is another compelling reason to proceed with evolving equilibria rather than an 
initial-value 3D resistive-MHD code; the former, with given profiles, can be checked (and upgraded, and redone, if need be) at any point in a time-dependent calculation and is moreover free from the possibility of small errors in the treatment of the rapid parallel transport showing up in the higher-order perpendicular tranport solution.

Such a coupled code would provide several additional capabilities:

- Beta-limits due to tearing modes.

- Self-consistent ripple transport; rotation and locked modes (for tokamaks and spheromaks).

- First transport code in the world (transport self-consistent with the magnetic geometry) for stellerators.

- In general, one could follow all of spheromak evolution except MHD events sufficiently fast that inertia is not negligible; if the formation phase (highly nonaxisymmetric) is evolving relaxed states, it too can be simulated using such a technique.

\section{References}

1. M. N. Rosenbluth and M. N. Bussac, "MHD Stability of Spheromak," Nucl. Fusion 19, 489 (1979).

2. J. H. Hammer, "MHD Tilting Modes for Nearly Spherical Compact Toroids with Arbitrary Plasma Pressure," Nucl. Fusion 21, 488 (1981).

3. J. M. Finn, W. M. Manheimer, and E. Ott, "Spheromak Tilting Instability in Cylindrical Geometry," Phys. Fluids 24, 1336 (1981).

4. J. M. Finn, W. M. Manheimer, and T. M. Antonsen, "Drift-Resistive Interchange and Tearing Modes in Cylindrical Geometry," Phys. Fluids 26, 962 (1983).

5. G. W. Hammett and W. M. Tang, "Kinetic and Resistive Effects on Interchange Instabilities for a Cylindrical Model Spheromak," Nucl. Fusion 23, 1503 (1983).

6. J. DeLucia, S. C. Jardin, and A. H. Glasser, "Resistive Stability of the Cylindrical Spheromak," Phys. Fluids 27, 1470 (1984).

7. M. Okabayashi and A. M. M. Todd, "A Numerical Study of MHD Equilibrium and Stability of the Spheromak," Nucl. Fusion 20, 571 (1980).

8. P. Gautier, R. Gruber, and F. Troyon, "Numerical Study of the Ideal-MHD Stability Limits in Oblate Spheromaks," Nucl. Fusion 21, 1399 (1981).

9. S. C. Jardin, "Ideal Magnetohydrodynamic Stability of the Spheromäk Configuration," Nucl. Fusion 22, 629 (1982). 
10. S. C. Jardin, M. S. Chance, R. L. Dewar, R. C. Grimm, and D. A. Monticello, "Tilting and Shifting Modes in a Spheromak," Nucl. Fusion 21, 1203 (1981).

11. S. C. Jardin and U. Christensen, "Stabilizing Windings for the Tilting and Shifting Modes in an Inductively Formed Spheromak," Nucl. Fusion 21, 1665 (1981).

12. C. Pfersich, R. Gruber, F. Troyon, "Free-Boundary MHD Stability of Pressureless Oblate Spheromaks - Dependence on Aspect Ratio and Elongation," Nucl. Fusion 23, 1127 (1983).

13. W. Park and S. C. Jardin, "Nonlinear Saturation of Nonresonant Internal Instabilities in a Straight Spheromak," Phys. Fluids 26, 1871 (1983).

14. W. W. Heidbrink, S. C. Jardin, and M. S. Chance, "Tearing-Mode Stability of a Forming Spheromak Plasma," Nucl. Fusion 22, 459 (1982).

15. J. DeLucia and S. C. Jardin, "Nonlinear Evolution of the Resistive Interchange Mode in the Cylindrical Spheromak," Phys. Fluids 27, 1773 (1984).

16. T. Hayashi and T. Sato, "Spheromak Global Instabilities and Stabilization by Nearby Conductors," Phys. Fluids 28, 3654 (1985).

17. S. O. Knox, Cris W. Barnes, G. J. Marklin, T. R. Jarboe, I. Henins, H. W. Hoida, and B. L. Wright, "Observations of Spheromak Equilibria Which Differ from the Minimum-Energy State and Have Internal Kink Distortions," Phys. Rev. Letters 56, 842 (1986).

18. R. Fitzpatrick, "The Effect of a Partial Resistive Shell on Magnetohydrodynamical Stability of Tokamak Plasmas," and references therein, draft in preparation for Nucl. Fusion (Dec., 1996).

19. S. W. Haney, L. D. Pearlstein, R. H. Bulmer, and J. P. Freidberg, "Vertical Stability Analysis of Tokamaks Using a Variational Procedure," to appear in Plasma Phys. Rep. (formerly Sov. J. of Plasma Phys.), (Sept., 1997).

20. J. B. Taylor, "Relaxation of Toroidal Plasma and Generation of Reverse Magnetic Fields," Phys. Rev. Letters 33, 1139 (1974).

21. J. B. Taylor, "Relaxation and Magnetic Reconnection in Plasmas," Rev. Mod. Physics 58, 741 (1986).

22. M. Yamada, N. Pomphrey, A. Morita, Y. Ono, and M. Katsurai, "Global Stability Study of the Ultralow Aspect Ratio Tokamak, ULART," Nucl. Fusion 36, 1210 (1996).

23. E. G. Zweibel and N. Pomphrey, "Tilt stability of rotating current rings with resistive conductors," Phys. Fluids 28, 2517 (1985).

24. M. N. Bussac, R. Pellat, D. Edery, and J. L. Soule, "Internal Kink Modes in 
Toroidal Plasmas with Circular Cross Sections," Phys. Rev. Letters 35, 1638 (1975).

25. L. E. Zakharov, "Internal Kink Mode in a Tokamak," Sov. J. Plasma Phys. 4, 503 (1979).

26. U. Shumlak and C. W. Hartman, "Sheared Flow Stabilization of the $m=1$ Kink Mode in $Z$ Pinches," Phys. Rev. Letters 75, 3285 (1995).

27. U. Shumlak, T. K. Fowler, and E. C. Morse, "Rotational Effects on the $m$ = 1 Magnetohydrodynamic Instability in Spheromaks," Phys. Plasmas 1, 643 (1994).

28. J. M. Greene and M. S. Chance, "The Second Region of Stability Against Ballooning Modes," Nucl. Fusion 21, 453 (1981).

29. T. H. Jensen, M.-S. Chu, and J. M. Greene, "Linear Stability of Force-Free Equilibria," Phys. Fluids 30, 2759 (1987).

30. Y. Kondoh, "Stable Equilibrium Configuration of Reversed-Field Pinch with the Partially Relaxed-State Condition," Nucl. Fusion 21, 1607 (1981).

31. J. W. Edenstrasser and W. Schuurman, "Axisymmetric Finite- $\beta$ Minimum Energy Equilibria of Weakly Toroidal Discharges," Phys. Fluids 26, 500 (1983).

32. A. Bhattacharjee, R. L. Dewar, and D. A. Monticello, "Energy Principle with Global Invariants for Toroidal Plasmas," Phys. Rev. Letters 45, 347 (1980); Phys. Rev. Letters 45 E, 1217 (1980).

33. L. Turner and J. P. Christiansen, "Incomplete Relaxation of Pinch Discharges," Phys. Fluids 24, 893 (1981).

34. A. Bhattacharjee and R. L. Dewar, "Energy Principle with Global Invariants," Phys. Fluids 25, 887 (1982).

35. R. N. Sudan, "Stability of Field-Reversed, Force-Free, Plasma Equilibria with Mass Flow," Phys. Rev. Letters 42, 1277 (1979).

36. E. Hameiri and J. H. Hammer, "Turbulent Relaxation of Compressible Plasmas," Phys. Fluids 25, 1855 (1982).

37. J. M. Finn and T. M. Antonsen, Jr., "Turbulent Relaxation of Compressible Plasmas with Flow," Phys. Fluids 26, 3540 (1983).

38. L. Turner, "Hall Effects on Magnetic Relaxation," IEEE Trans. Plasma Sci. 14, 849 (1986).

39. K. Avinash, "Some Stationary Solutions of Two-Fluid Magnetohydrodynamic Equations," Phys. Fluids B 4, 3856 (1992).

40. L. Woltjer, "A Theorem on Force-Free Magnetic Fields," Proc. Natl. Acad. Sci. USA 44, 489 (1958); "On Hydromagnetic Equilibrium," 44, 833 (1958); 
"Hydromagnetic Equilibrium II. Stability in the Variational Formulation," 45, 769 (1959); and "The Stability of Force-Free Magnetic Fields," Astrophys. J. 128, 384 (1958).

41. L. C. Steinhauer and A. Ishida, "Finite- $\beta$ Minimum Energy States of a TwoFluid Flowing Plasma," submitted to Phys. Plasmas (1996).

42. J. W. Edenstrasser, "The Plasma Transport Equations Derived by Multiple Time-Scale Expansions and Turbulent Transport. I.," Phys. Plasmas 2, 1192 (1995); J. W. Edenstrasser and M. M. M. Kassab, "The Plasma Transport Equations Derived by Multiple Time-Scale Expansions. II.," Phys. Plasmas 2, 1206 (1995).

43. M. N. Rosenbluth and P. H. Rutherford, "Tokamak Plasma Stability," in Fusion, E. Teller, ed., Vol. 1, Part A, 80 (1981).

44. F. J. Wysocki, J. C. Fernández, I. Henins, T. R. Jarboe, and G. J. Marklin, "Evidence for a Pressure-Driven Instability in the CTX Spheromak," Phys. Rev. Letters 21, 2457 (1988).

45. A. H. Reiman, "Tokamak magnetic islands in the presence of nonaxisymmetric perturbations," Phys. Fluids B 3, 2617 (1991); A. Reiman and D. Monticello, "Tokamak error fields and locked modes," Phys. Fluids B 3, 2230 (1991). 


\section{Spheromak Dynamo}

\subsection{Basic description of the dynamo in relaxed-state devices}

This section describes the basics of dynamo theory, and the application to relaxed state devices.

Dynamo fluctuations are generally present in any relaxed-state device with resistivity. This includes spheromaks and reversed field pinches (RFPs). In general terms, the term dynamo refers to the process of magnetic field generation by electrically conducting fluids. In relaxed-state devices, a dynamo arises as follows.

First, according to the Woltjer/Taylor relaxation theory, ${ }^{1,2}$ plasma equilibrium tends to relax toward a state in which magnetic field energy is minimized, subject to the constraints of fixed helicity and magnetic flux. A simple variational calculation applied to this principle produces the following equation for the magnetic field:

$$
\nabla \times \mathbf{B}_{0}=\mu \mathbf{B}_{0}
$$

This equation, along with specification of boundary conditions and the total magnetic helicity and flux in the plasma, gives the magnetic field toward which the plasma tends to evolve. This postulate is generally borne out by observations on relaxed-state devices such as the spheromak or reversed field pinch:

Second, in the devices under consideration, the averaged magnetic field evolves by the averaged induction equation:

$$
\frac{\partial}{\partial t} \mathbf{B}_{0}+\frac{1}{c}\langle\widetilde{V} \times \widetilde{B}\rangle=\frac{\eta c^{2}}{4 \pi} \nabla^{2} \mathbf{B}_{0}+\text { external drive }
$$

where the subscript () refers to some averaging procedure (such as ensemble or time averaging), $\sim$ refers to deviations from the average, and we take $V_{0}=0$ for simplicity. The external drive refers to externally supplied electric fields, such as given by an inductive core or a Marshall gun. Thus, the magnetic field evolves under the influence of fluctuations, resistive decay, and external drive, respectively.

The important point is that unless the resistive decay and external drive add precisely zero, $\mathbf{B}_{0}$ is driven away from the minimum energy configuration. As this occurs, excess magnetic field energy (i.e., free energy, usually in the form of a gradient in the parameter $\lambda=\mathbf{B}_{0} \cdot \nabla \times \mathbf{B}_{0} / B_{0}^{2}$ builds up in the plasma, and becomes available to destabilize fluctuations, $\widetilde{V}$ and $\widetilde{B}$. The fluctuations grow, and couple nonlinearly. In broad terms, the fluctuations stir up the plasma, and 
allow relaxation back toward the minimum energy state, thus compensating for the imperfect balance between drive and dissipation.

An more detailed analysis of the turbulent relaxation can be given can be given in terms of spectral transfer dynamics, as follows. Although the precise details of the turbulence are generally quite complicated, two quantities are conserved under the constraints of ideal MHD: magnetic helicity and energy. Given these two invariants, a situation of bi-directional spectral transfer tends to ensue: the spectral helicity energy tends to evolve toward longer wavelengths, and energy toward shorter. ${ }^{3,4}$ The inversely transfered helicity eventually joins the averaged field, where it compensates the imbalance mentioned above. The forwardly transferred energy eventually reaches dissipative scales, where it is transferred to electron or ion heat. ${ }^{5}$ The inverse transfer of helicity is subject, of course, to the constraint that it must carry with it at least the minimum amount of energy as specified by the boundary conditions. The forward energy transfer has a similar constraint, but in the limit where the transfer continues to very long wavelengths before dissipating, the lost helicity is negligible. In this way, the spectral transfer characteristics of the turbulence allow relaxation back to the minimum energy state.

It is also possible to give a dynamical description of the dynamo, in terms of the detailed forces generated by the unstable fluctuations. This has been undertaken for the RFP, both theoretically ${ }^{6,78}$ and numerically., ${ }^{9,10}$ In short, these studies have generally found that a current profile peaked on the magnetic axis can destabilize $m=1$ tearing modes, which then can act on the equilibrium field in two ways. The first is the direct quasilinear production of a dynamo field, which damps current near the magnetic axis, and drives field relaxation, as required to restore the relaxed state. The second is the coupling of an $m=1, n=n_{0}$ mode with an $m=1, n=$ $n_{0}+1$ mode, which drive a $m=0, n=1$ mode, which in turn quasilinearly drives an equilibrium dynamo field, again in the direction required for relaxation. Both of. these effects are consistent with the energetics picture given above, sacrificing some generality but offering more detail.

The dynamo effect is beneficial to a spheromak, in that it gives a method to drive current on the magnetic axis with a source on the edge. However, there is an elementary argument, known as Cowling's theorem, ${ }^{11}$ which shows that dynamo fluctuations cannot be axisymmetric. The upshot of this is that the dynamo fluctuations break magnetic surfaces, and connect the core plasma with the edge plasma, allowing loss of heat and particles on fast parallel time scales. While the details of the transport are complicated and understanding is still emerging, it is generally 
agreed that reducing the amplitude of dynamo fluctuations has the potential of greatly improving spheromak confinement.

Present-day dynamo theory for a spheromak is at a very primitive stage. On the other hand, the theory of the RFP dynamo is considerably more evolved [see Ref. 10], and can be used as a model. Four important differences should be mentioned. First, the $\lambda$ profile of the RFP is generally peaked on the magnetic axis, while that of the spheromak is peaked on the edge (at least during the sustainment phase, as described below). This means that the dynamo tends to act in opposite directions on each. The second difference is that the RFP has a much larger aspect ratio, and much theoretical progress can be made with a cylindrical model. On the other hand, the spheromak has an aspect ratio close to 1 , and the cylindrical model is much less applicable, making theoretical progress somewhat more difficult. A third important difference is the prominence of open field lines in the spheromak. That is, in the RFP reconnection dynamics occur almost entirely in regions of closed field lines, whereas in the spheromak, the central column where the current is driven is largely reconnected to the wall. Fourth, in the RFP, helicity flows from a low resistivity region to high (core to edge), while the spheromak is opposite. This means that the spheromak dynamo is aided by classical forces [namely the thermal instability, where currents tends to peak in low resistivity regions], while the RFP dynamo opposes them. In this sense, the spheromak dynamo could possibly be less destructive, as it may require less flow for relaxation.

\subsection{Dynamo in a spheromak plasma}

A spheromak discharge may be divided into three phases relative to the dynamo.

1. Formation phase: A spheromak discharge is initially formed with some unrelaxed current profile, and the large surplus of free energy allows a number of current driven MHD instabilities, both ideal and resistive. The unstable fluc-tuations grow, and if they are not so severe as to destroy the plasma, saturate nonlinearly, and then proceed to dissipate the excess energy, while conserving helicity. The dual transfer scenario described above shows how this occurs. As the energy is dissipated the fluctuations reduce in amplitude, leaving the plasma in a more quiescent and approximately relaxed state. This point is the end of the formation phase.

2. Sustainment phase: In this phase, the current is driven at the outer radii

of the spheromak, causing the $\lambda=\mathbf{B}_{0} \cdot \nabla \times \mathbf{B}_{0} / B_{0}^{2}$ to have a "hollow" profile relative to the magnetic axis. The forces of the dynamo, which tend to flatten 
$\lambda$, generally damp the current on the edge and drive it on the magnetic axis.

3. Decay phase: The decay phase begins when the current drive at the outer radii is switched off, and the plasma current is allowed to decay. This causes $\lambda$ to be peaked on the magnetic axis. The action of the dynamo in this case is to damp current on the magnetic axis and drive it at the outer radii. For this reason, the spheromak dynamo most resembles that of an RFP in this phase.

In each of the three phases, one would like to understand the unstable dynamo modes, the nature of the nonlinear coupling between the modes, and the heat and particle transport resulting from the fluctuations. For the Livermore Sustained Spheromak Physics Experiment, the sustainment phase holds the greatest interest. This also happens to be the phase of least detailed knowledge, both because this phase has been short in previous spheromak experiments, and because of the inverted $\lambda$ profile, it least resembles an RFP.

Some components of a phenomenological picture can be gathered from recent experiments on the SPHEX spheromak, ${ }^{12}$ and from similarities with an RFP. The SPHEX group reported two kinds of dynamo fluctuations: single mode and turbulent. The single mode is an $n=1$ oscillation concentrated at the geometric axis $(R \sim 0)$ which gives an electric field opposing the applied current. The turbulent fluctuations were located in the "annulus" (closed flux surface region) and give an electric field in the same direction as the applied current. These observations suggest the dynamo is initiated by a coherent $n=1$ kinking of the central column, which then reconnects with the "annulus" region of the plasma. In the reconnection process, the kink mode will stir up the field lines in the annular region, causing braiding and stochasticity which will propagate toward the magnetic axis. This is illustrated in Fig 2. Alternatively viewed, the energy of the kink mode cascades to different wavelengths, and the accompanying magnetic stochasticity allows current to flow freely between surfaces, allowing flattening of the $\lambda$ profile.

This phenomenological picture is reminiscent of the RFP result that there are two coexistent dynamos: the discrete dynamo, with a few coherent modes and well separated growth and crash phases, and the continuous dynamo, with steady-state saturated turbulence. ${ }^{13}$ One difference though is that in the spheromak the two dynamos occupy separate spatial locales, while this is apparently not observed in the RFP. 


\subsection{Proposed course of study for the spheromak dynamo}

\section{a. Theory and phenomenology}

There are a number of interesting directions to pursue in establishing the theory of the spheromak dynamo. The following is organized starting with the more established methodologies (i.e., linear theory), proceeding to the more speculative.

The most obvious problem is establishing the linear instability theory of the "dynamo modes," that is, the first modes to become unstable as the spheromak equilibrium decays away from a fully relaxed configuration. We focus on the sustainment phase, both because this is of most interest to the Livermore Sustained Spheromak Physics Experiment, and because the dynamo during the decay phase may, to lowest approximation, be assumed to resemble that of an RFP.

The geometry is shown in Fig. 1. There is a central geometric axis carrying current driven by the gun, which is surrounded by an annular region carrying the internal spheromak current. At the crudest level, the dynamo instability might occur along the following lines. The plasma in the neighborhood of the geometric axis experiences two magnetic fields: that from itself, which is roughly horizontal and we call $B_{h}$, and that from the annular region, which is roughly vertical and we call $B_{v}$. Very roughly speaking, such configurations tend to become unstable when $B_{h}^{2} / B_{v}^{2}$ exceeds some critical value, ${ }^{14} c_{c r i t}$

$$
\frac{B_{h}^{2}}{B_{v}^{2}} \gtrsim c_{c r i t} .
$$

If we estimate that $B_{h} \propto I_{g u n} / r_{c e n t}$, where $I_{g u n}$ is the total current through the gun and $r_{c e n t}$ is the radius of the central column, and that $B_{v} \propto I_{p} / a$, where $I_{p}$ is the total current carried by the annular region and $a$ is the radius of the annular region, then the above becomes:

$$
\frac{I_{g u n}^{2} a^{2}}{I_{p}^{2} r_{c e n t}^{2}} \gtrsim c_{c r i t}
$$

which gives a rough idea of when the geometric axis becomes unstable, as shown in Fig. 2. This instability can also be viewed as a flattening of the $\lambda$ profile. Estimating the current density on the geometric axis as $J_{g a} \sim I_{g u n} / r_{\text {cent }}^{2}$, current density on the magnetic axis as $J_{m a} \sim I_{p} / a^{2}$, the field on the geometric axis as $B_{g a}=B_{v} \sim I_{p} / a$, and the field on the magnetic axis as $B_{g a} \sim I_{g} u n / a$, then Eq. (4.4) can be written as

$$
\frac{J_{g a}}{B_{g a}} \frac{B_{m a}}{J_{m a}}=\frac{\lambda_{g a}}{\lambda_{m a}} \gtrsim c_{c r i t} .
$$


Equation (4.5) indicates that the instability proceeds when the $\lambda$ profile becomes sufficiently peaked on the geometric axis. This demonstrates the important feature that this instability can proceed regardless of the ratio of total currents carried in the two regions; it is more a matter of.current density. More detail in this instability criterion is obviously desirable. One would like to include a number of effects, including fuller geometry of the plasma, a consideration of the motion of the closed flux surface region, boundary conditions on the gun and lower wall, separation of the plasma on the geometric axis into regions where the lines tie to the wall and carry all the way to the gun.

The next question which arises is the method of reconnection between the geometric column and the core plasma. This depends on the detailed nature of the above instability, but one possibility is forced reconnection ${ }^{15}$ of the geometric column driven into the annular region (Fig. 2).

The dynamo in the the annular region is another important issue. The SPHEX observations suggest that these fluctuations are composed of high $n$ turbulence, which may lead one to believe that linear stability theory may not be relevant. To avoid addressing the precise details of the field line braiding and precise nature of the high $k$ modes, which can be complicated, one can cover this in terms of strong turbulence approach adopted in Ref. 5 , in which the turbulence is seen as a dual cascade of energy and helicity, injected into the spectrum by the $n=1$ kind mode. This approach allows several key issues to be addressed, such quantification of the forward and inversely cascaded energy and helicity, the spectral falloff rate of the magnetic fluctuations, ${ }^{16}$ and how the forward cascaded energy is eventually dissipated. A key issue which can be addressed by this approach is the efficiency of this phase of the dynamo. The dynamo fluctuations inevitably contain an excess amount of energy in addition to what is required to drive the relaxed field, which must be dissipated somehow. This reduces the efficiency with which the external. drive supplies current to the annular region. Note that this loss of efficiency is in addition to that discussed by Barnes et al. ${ }^{17}$

Another issue that can be addressed with existing approaches is the scaling of $\widetilde{B}$ with the Lundquist number, $S=4 \pi a v_{A} / \eta c^{2}=$ (resistive time)/(ideal time), (where $a$ is an equilibrium scale length, $v_{A}$ is the Alfvén velocity, and $\eta$ is the resistivity). There has been recent theoretical, ${ }^{18}$ experimental, ${ }^{19}$ and numerical ${ }^{20,21}$ work on this topic, with some degree of agreement in all. The main issue is how much $\widetilde{B}_{r m}$ can be expected to reduce with decreasing resistivity; this is discussed further below.

One final issue we will mention here is the current profile in incompletely relaxed 
spheromak. The difference between a fully relaxed profile and actual profiles can be large, and such departures can have important implications for pressure driven instabilities. This could borrow from parallel work in the RFP. Recently, Kadomtsev has noted ${ }^{22}$ that the current profile in an RFP seems to be midway between the ideally relaxed Taylor State, and an older paramagnetic model, which balances the parallel force from toroidal $E$ in the presence of a non-toroidal $B$. This suggests a straightforward study for the present case: how does this average come out on a spheromak? The Taylor state for a spheromak is well known. ${ }^{23}$ The paramagnetic state in general is obtained by assuming that Ohm's law applies, but only for the parallel component of $\mathbf{J}_{\mathbf{0}}$. The perpendicular component of $\mathbf{J}$ is taken to be zero, under a force-free assumption. This gives

$$
\eta \mathbf{J}_{0}=\hat{b} \hat{b} \cdot \mathbf{E}_{0},
$$

where $\hat{b}=\mathbf{B}_{0} / B_{0}$. This, together with Ampère's law and a prescription for the applied electric field $\mathbf{E}_{0}$ yield a solution for the paramagnetic $\mathbf{B}_{0}$. The average of these two fields could provide some idea of the true current profile to be expected in a spheromak.

\section{b. Examining means of reducing dynamo fluctuations}

The second part of the dynamo study is examining means of reducing dynamo fluctuations. This is a very important topic, and provides the true motivation for all the above study. The group at Madison Symmetric Torus (MST) is currently testing several ways to quench dynamo turbulence on the RFP. We hope to pursue analogous lines with the Livermore spheromak.

We consider three approaches: rely on " $S$-scaling," (in which magnetic turbulence may naturally reduce at the high temperatures of a reactor), feedback stabilization, and profile control.

1. The $S$ scaling approach requires that $\alpha$ in the formula $\langle\tilde{B}\rangle_{r m s} \propto S^{-\alpha}$ is large enough. If $\alpha \gtrsim 1 / 2$ or better, then transport in reactor regimes is predicted to be adequately small, ${ }^{24}$ and indeed some experimental evidence ${ }^{25,26}$ supports $\alpha \sim 1 / 2$ However, more recent studies ${ }^{18,19,20,21}$ suggest that for $S$ closer to reactor regimes, $\alpha$ is more in the range of 0 to $1 / 4$. However these scalings were based on RFP plasmas, and it is quite possible that a spheromak could behave differently. Therefore we intend to continue these studies for the Livermore spheromak, but at the same time, examine additional means of reducing dynamo fluctuations. 
2. A second way is feedback stabilization of dynamo modes. For example, in a tokamak, ECRH applied just outside the mode rational surface flattens the temperature and thus the current profile, stabilizing the mode. The advantage is that this requires relatively small amounts of RF power. The disadvantage is that stabilizing some particular dynamo modes still leaves the basic problem: the current profiles will continue to evolve away from the minimum energy state, until the next dynamo mode becomes unstable. The result may be even more catastrophic than before, since it will entail release of larger amounts of energy.

3. The third method is current drive. Here current is somehow driven in the closest possible approximation to the relaxed profile, so that the equilibrium doesn't stray far from relaxation, making relaxation events small. In a spheromak at the crudest level, driving current in the toroidal direction at $r=0$ could compliment the poloidal current driven at the edge. This is different from RFP current drive methods in at least one important way. In the RFP, the current is driven naturally on the magnetic axis; thus there must be an auxiliary current drive at the edge. In the spheromak, current is generally driven externally from the edge; thus generally there must be auxiliary current drive from the core. There are a few methods which we will examine, which we will mention here only briefly, as they are all quite speculative at this stage. First, there are several types of radio frequency (RF) current drive available, although these have the problem being that they are generally expensive. A second possible method is to inject helicity into the core of the spheromak via the use of compact toroids (CTs) driven from some gun at the plasma edge, thereby feeding the large spheromak with smaller ones. A third possibility is some form of inductive drive, similar to a tokamak. The S-1 device at Princeton ${ }^{27}$ accomplished this via a flux core encircling the plasma, and with a poloidal flux transformer on the geometric axis (although this somewhat defeats the purpose of having a simple plasma with a minumum of external circuitry). Recently, it has been suggested by J. M. Finn ${ }^{28}$ that it may be possible to use the plasma surrounding the geometric axis as a flux core, to some extent, changing the magnetic field on axis to induce a toroidal electric field on the magnetic axis. In the RFP, it has been suggested ${ }^{29}$ that applying out-of-phase oscillating voltages to the poloidal and toroidal circuits (" $F-\Theta$ pumping") can be used to drive a mean toroidal voltage. Janos and Yamada ${ }^{27}$ have suggested that this technique may be applied to a spheromak, by oscillating the currents 
in the poloidal and toroidal field coils within an external flux core. It may also be possible to devise a similar technique by oscillating the field and current supplied across the geometric axis of the spheromak. 


\section{References}

${ }^{1}$ L. Woltjer, Proc. Natl. Acad. Sci. USA, 44, 489 (1958).

${ }^{2}$ J. B. Taylor, Rev. Mod. Phys, 58, 741 (1986).

${ }^{3}$ U. Frisch, A. Pouquet, J. Leorat, and A. Mazure, J. Fluid Mech. 68, 769 (1975).

${ }^{4}$ A. Pouquet, U. Frisch, J. Leorat, J. Fluid Mech. 77, 321 (1976).

${ }^{5}$ N. Mattor, P. W. Terry, and S. C. Prager, Comments on Plasma Physics 15, 65 (1992).

${ }^{6} \mathrm{H}$. R. Strauss, Phys. Fluids 28, 2786 (1985).

${ }^{7}$ E. Hamieri and A. Bhattacharjee, Phys. Fluids 30, 1743 (1987).

${ }^{8}$ J. H. Holmes, B. A. Carreras, P. H. Diamond, and V. E. Lynch, Phys. Fluids 31, 1166 (1988).

${ }^{9}$ Y. L. Ho and G. G. Craddock, Phys, Fluids B 3, 721 (1991).

${ }^{10}$ D. D. Schnack, in Physics of Alternative Magnetic Confinement Schemes, ed. by

S. Ortolani and E. Sindoni, SIF, Bologna (1991).

${ }^{11} \mathrm{H}$. K. Moffatt, Magnetic field generation in electrically conducting fluids, (c)1978, Cambridge University Press, p. 113.

${ }^{12}$ A. al-Karkhy et al., Phys. Rev. Lett. 70, 1814 (1993).

${ }^{13}$ B. Chapman, Phys. Plasmas 3, March (1996).

14 J. D. Jackson, Classical Electrodynamics, 2nd edition, p. 483 (1975).

${ }^{15} \mathrm{H}$. E. Petschek in AAS-NASA Symposium on the Physics of Solar Flares, edited by W. N. Hess (NASA, Washington, D. C.), NASA SP-50, p. 425 (1965)

${ }^{16} \mathrm{P}$. W. Terry, Phys. Plasmas 1, (1994).

${ }^{17}$ Cris W. Barnes et al., Phys. Fluids 29, 3415 (1986).

${ }^{18}$ N. Mattor Phys. Plasmas 3, 1578 (1996).

${ }^{19}$ M. R. Stoneking, J. S. Sarf, C. R. Sovinec, and N. Mattor Bull. Am. Phys. Soc. (1996).

${ }^{20}$ C. R. Sovinec, Magnetohydrodynamic Simulations of Noninductive Helicity Injection in the Reversed-Field Pinch and Tokamak, $\mathrm{PhD}$ Thesis, UIniv. Wisconsin, Madison (1995).

${ }^{21} \mathrm{~S}$. Capello and D. Biskamp, in Proceedings of the 20th European Conference on Plasma Physics and Controlled Fusion and Plasma Heating, (European Physical Society, Petit-Lancy, Switzerland, 1993) Vol. II, 3-13 (1993).

${ }^{22}$ B. B. Kadomtsev, Comments on Plasma Physics and Controlled Fusion, 15, 211 (1993).

${ }^{23}$ M. N. Rosenbluth and M. N. Bussac, Nucl. Fusion 19, 489 (1979). 
${ }^{24}$ T. K. Fowler, Heat Loss by Helicity Injection in Spheromaks, UCRL-ID-116975 (1994).

${ }^{25}$ R. J. LaHaye et al., Phys. Fluids 27, 2576 (1984).

${ }^{26}$ E. B. Hooper, J. H. Hammer, C. W. Barnes, J. C. Fernandez, and F. J. Wysocki, A Reexamination of Spheromak Experiments and Opportunities, Fusion Technol., March, 1996.

${ }^{27}$ Alan C. Janos and Masaaki Yamada, Fusion Technology 9, 58 (1986).

${ }^{28}$ John M. Finn, personal communication.

${ }^{29}$ M. K. Bevir and J. W. Gray in Proceedings of the Reverse Field Pinch Theory Workshop, edited by H. R. Lewis and R. A. Gerwin (Los Alamos National Laboratory, Low Alamos, New Mexico), Session III, paper A-3 (1980). 


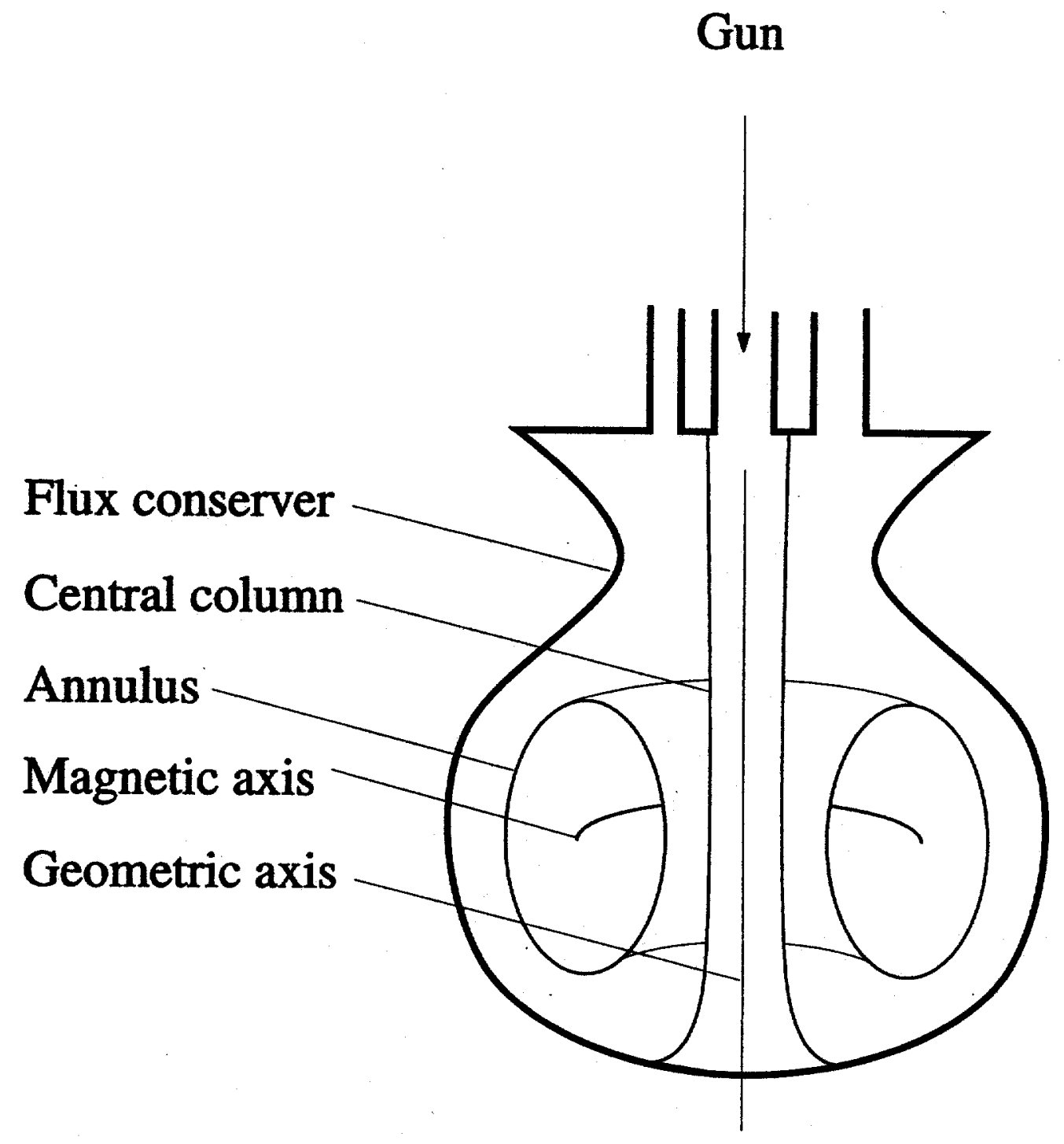

\section{Figure 1}

Structure of spheromak 


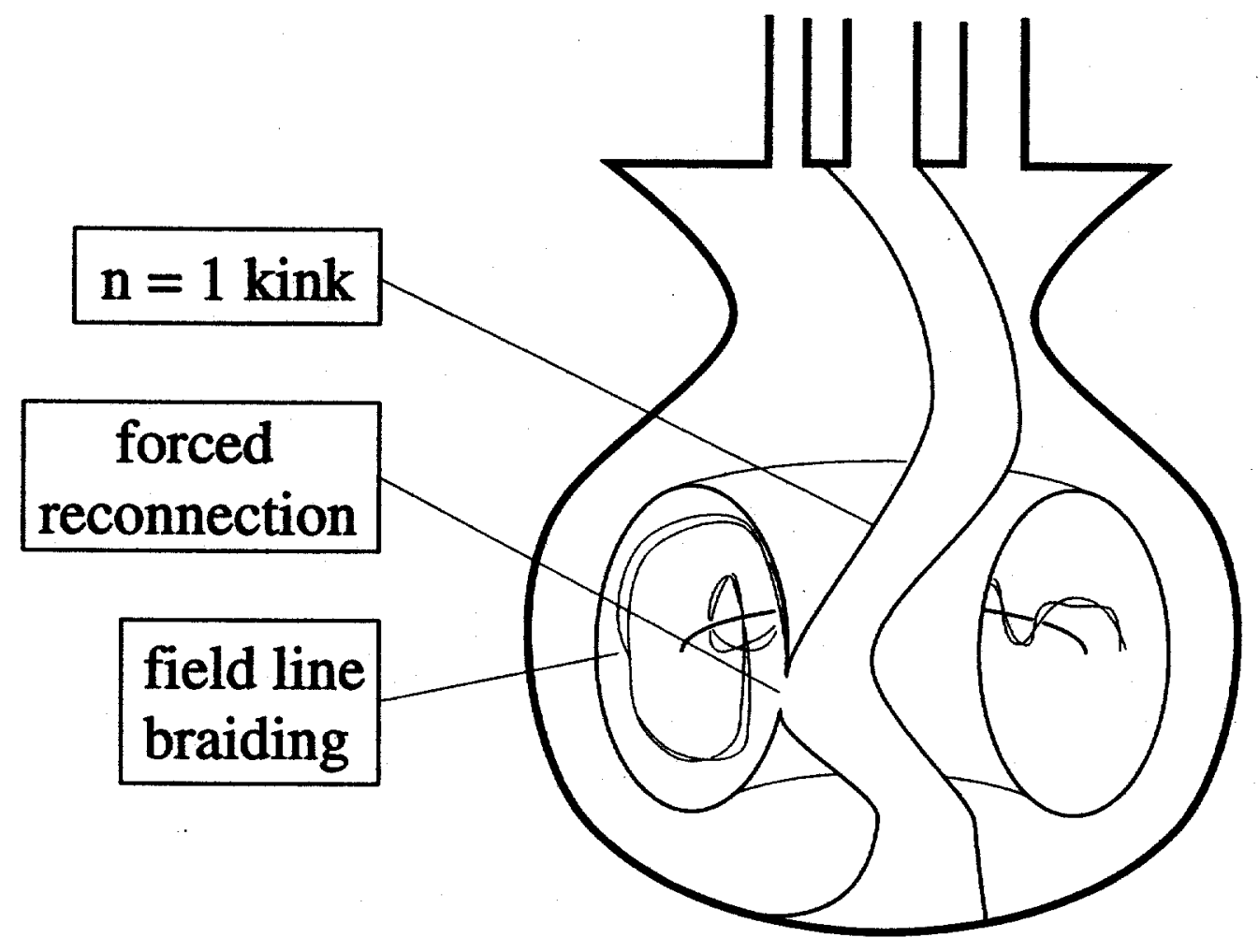

Figure 2

Dynamo fluctuations 


\section{Edge Plasma in Spheromaks}

\subsection{Introduction}

The plasma in spheromaks, as well as in other fusion devices, is surrounded by cold walls. The processes occurring in the boundary layer between the plasma and the walls determine many phenomena important for the overall performance of the spheromak. Among them are: current flow outside the separatrix (i.e., in the region where the magnetic field lines "begin" and "end" on the walls); generation of impurities and their penetration to the core; specific instabilities which may affect transport in the transition region, etc. Related to these issues is the issue of the magnetic field errors: as the transition layer is narrow, it is sensitive even to minor field errors.

We will discuss the edge plasma physics in the parametric domain characteristic of the Livermore Spheromak. We will concentrate our discussion on the plasma behavior on the open field lines, beyond the separatrix. We will consider only a quasi-steady-state phase of discharge, after the spheromak configuration has already been formed in the flux conserver.

Based on past experience, one can expect that the plasma parameters in the immediate vicinity of the separatrix will be:

$$
n=10^{14} \mathrm{~cm}^{-3}, T=20 \mathrm{eV}, Z_{e f}=2 .
$$

These parameters will be used only for a general guidance; in specific cases where we try to make "self-consistent" estimates, the results may somewhat differ from Eq. (1) but, as it turns out, generally this difference is not very large. The definition of $Z_{\text {eff }}$ that we are using is: $Z_{e f f}=\Sigma n_{\alpha} Z^{2} \alpha / \Sigma n_{\alpha}$.

With regard to the poloidal current density, we assume the value $-1.5 \mathrm{kA} / \mathrm{cm}^{2}$ consistent with the model of MHD equilibrium discussed in Sec. 1 of this report. Typical magnetic field strength will be taken to be $-1.5-2 \mathrm{~T}$.

As a source of formulas for transport coefficients and radiation processes we are using Braginski's survey' and the NRL Plasma Physics Formulary. ${ }^{2}$

\subsection{The role of skin effect in the flux conserver}

The conductivity of copper at room temperature is $\sigma_{\text {copper }}-5.3 \cdot 10^{17} \mathrm{~s}^{-1}$ and corresponds to the magnetic diffusivity 


$$
D_{m} \equiv c^{2} / 4 \pi \sigma_{\text {copper }}=150 \mathrm{~cm}^{2} / \mathrm{s} .
$$

For the two-millisecond pulse, the skin depth,

$$
\delta \sim\left(2 D_{m} t\right)^{1 / 2},
$$

is $\sim 8 \mathrm{~mm}$. The radius of curvature of the flux-conserver surface is everywhere considerably larger than $8 \mathrm{~mm}$. Therefore, every element of the surface can be considered as planar with respect to the skin effect. The amount of the poloidal flux "soaked" into the surface is therefore directly related to the magnetic field strength $B_{p}$ in the particular point of the flux conserver and is approximately equal to

$$
2 \pi r B_{p} \delta
$$

where $r$ is the toroidal radius of the corresponding point.

Let us analyze the consequences of this observation for those field lines which are passing near the magnetic axis. Obviously, some bunch of them will be intercepted by the flux conserver near the point " $A$ " in Fig. 1a. Therefore, near the plasma axis, there exists a flux tube which is not connected with the electrodes of the plasma gun: the current flows directly between the upper and the lower plates of the flux conserver. The radius $a$ of this flux tube can be evaluated from magnetic flux conservation. Assuming that the magnetic field near the points $O$ and $A$ in Fig. $1 \mathrm{a}$ are of the same order of magnitude, one finds that

$$
a \sim(2 R \delta)^{1 / 2}
$$

For $R=25 \mathrm{~cm}$, one finds that $a \sim 6 \mathrm{~cm}$. At a current density $\sim 1.5 \mathrm{kA} / \mathrm{cm}^{2}$, the total poloidal current through this column will be $\sim 150 \mathrm{kA}$. This part of the current is sensitive to the conditions on the upper and lower plates (not in the gun!). In particular, this current would be strongly reduced by the replacement of the solid flux conserver by a flux conserver made of mesh. A strong effect caused by such a replacement in earlier spheromak experiments may point out at the importance of the current near the axis for the overall performance of the spheromak.

The presence of the inevitable poloidal variation of the magnetic field on the outer surface of the flux conserver may cause the appearance of field lines of the type shown in Fig. 5.1 b, i.e., beginning and ending on the same electrode. We show in Sec. 5.6 that the main heat source on the open field lines is Joule heating, with only a minimum contribution of the heat flux from the hot plasma core. Clearly, at the field lines of the type shown in Fig. $5.1 \mathrm{~b}$ there is no voltage applied between the "ends" of the field line and the Joule dissipation is negligible. On the other hand, energy sinks (which are predominantly determined by parallel thermal conductivity to the walls) are still present on these field lines. Accordingly, one can expect that the plasma temperature on such field lines will be 
much lower than on field lines connecting electrodes of the opposite polarity. It may happen that the temperature will be so low that even the ionization degree on these field lines will be low.

In decaying spheromaks in mesh (open) flux conservers, ${ }^{3}$ substantial energy loss occurred on edge field lines. Field errors were large, with $25 \%$ of the field lines estimated to intersect the metal surface. The helicity transport to these lines was sufficient to generate an effective electric field large enough to maintain ionization. The resulting field was at the Paschen breakdown limit for hydrogen, thus maintaining plasma on the open field lines, and dominated the ohmic energy dissipation during the decay. Magnetic field errors reduced the fieldline length, thus increasing the effective electric field at the Paschen condition. As a result, edge ohmic dissipation was increased sigificantly in the decaying spheromak. ${ }^{3}$ In a solid flux conserver with low magnetic errors, the energy decay was found to depend on the core electron temperature, ${ }^{4}$ and the edge losses did not dominate the power balance. Fieldline errors can thus interfere with successful operation of the spheromak, and although their effects are probably not dominant during sustainment, careful design to minimize them is important. Similar requirements are known to be important for RFPs. ${ }^{5}$

Dynamo activity may induce fluctuations of the magnetic field and, therefore, the appearance of a pulsating loop voltage on the field lines of the type shown in Fig. 1b. The corresponding pulsating currents will serve as a source of plasma heating on these field lines. Therefore, plasma temperature on such field lines will be a direct measure of the dynamo activity near the plasma edge.

In our discussion of the skin effect, we implied that the initial magnetic field does not intersect the surface of the upper and lower electrodes. Then, the flux intercepted by the electrodes is, indeed, determined by the skin effect. One may consider a deliberate creation of a bias magnetic field that would intersect the surfaces of the upper and/or lower electrodes. This field may be directed in the same sense as the on-axis field of the spheromak, or in the opposite direction. In the latter case, additional X-point(s) will be formed near the axis. One may conceive the situation shown in Fig. 5.1c, where parts of the upper and lower electrodes are "shielded" by the magnetic field (this can be achieved by generating the properly shaped bias magnetic field). To prevent the current from flowing across the field lines, the magnetic shield should be thicker than the region where the plasma is cold and un-magnetized. Whether this type of arrangement will improve performance of the machine is an open question. What we want to emphasize here is that this might be a convenient way of controlling the current flow between the upper and lower electrodes. 


\subsection{Plasma collisionality}

At the parameters given above, the mean free path of plasma particles on the open field lines is short: $\lambda_{e i}-\lambda_{i i} \sim 6 \mathrm{~cm}$. A typical connection length will be probably $2-3 \mathrm{~m}$; in other words, the collisionality is strong enough to make effects of non-local transport negligible. (This conclusion may change if the plasma temperature is, in fact, higher than $20 \mathrm{eV}$, but such high values are not very probable, see below). Electron-electron and ion-ion collision times are by orders of magnitude shorter than the duration of the pulse. Even the electronion energy exchange time is short $\left(-2 \cdot 10^{-4} \mathrm{~s}\right.$ for the energy exchange between the electrons and deuterons). Parenthetically, one can mention that for the plasma core, at the nominal temperature of $400 \mathrm{eV}$, the collisional energy exchange time is longer than the pulse length.

\subsection{Current flow on the open field lines}

The relative velocity $u$ of electrons and ions is determined by the current density $j$ and plasma density $n$ :

$$
u(\mathrm{~cm} / \mathrm{s})=6 \cdot 10^{18} j\left(\mathrm{~A} / \mathrm{cm}^{2}\right) / n\left(\mathrm{~cm}^{-3}\right) .
$$

In the standard mode of operation, the current density on the open field lines is expected to be in the range of $1.5 \mathrm{kA} / \mathrm{cm}^{2}$. This corresponds to $u$ of order of $10^{8} \mathrm{~cm} / \mathrm{s}$, much greater than the ion thermal velocity, $v_{\pi}=5 \cdot 10^{6} \mathrm{~cm} / \mathrm{s}$ (deuterium, $20 \mathrm{eV}$ ), and comparable with the electron thermal velocity, $v_{T_{e}}=2 \cdot 10^{8} \mathrm{~cm} / \mathrm{s}$.

If generation of electrons on the walls (via the secondary and photo-emission) is small, then the parallel (to the magnetic field) current density cannot exceed the ion saturation current, which is of order of $e n v_{T}$, and the plasma would not be able to sustain the required current on the open field lines. On the other hand, in the previous spheromak experiments, no obvious limitations on the current have been noticed. Therefore, one has to assume that there is either a strong electron emission from the walls, or the plasma density near the walls is considerably higher than in the rest of the open field line region, or both. Our present understanding is that dense cold plasma near the walls will be formed almost inevitably (see below). The presence of the dense plasma near the electrodes may provide conditions where the current in the central (low density) part of the flux tube is carried by the electrons, while near the electrodes the current does not exceed the ion saturation current (because of a high plasma density there). 
Emission of electrons from the walls can occur via the photo-effect (under the action of the $10-20 \mathrm{eV}$ photons emitted by the plasma) and via thermo-ionic and field emission from the micro-spikes and other microstructures. Note that the intersection angle of the field lines with the flux conserver is small $2 \delta r \sim 0.06$. Accordingly, at a parallel (to the magnetic field) current density $\sim 1.5 \mathrm{kA} / \mathrm{cm}^{2}$, the normal component of the current density on the wall is $\sim 80 \mathrm{~A} / \mathrm{cm}^{2}$. At the expected intensity of the radiative losses, such a current can probably be maintained even by photo-emission alone.

In the plasma gun, the field lines intersect its surface also at a small angle. This also helps to keep the required normal component of the current density on the electrode at the reasonable level $\sim 100 \mathrm{~A} / \mathrm{cm}^{2}$. However, the whole issue of current generation at the electrodes is not very clear now and we are going to return to it in the future.

\subsection{Longitudinal pressure equilibrium}

For the connection length

$$
L-2-3 \mathrm{~m}
$$

the acoustic time $U c_{s}$ is of the order of $10^{-4} \mathrm{~s}$, considerably shorter than the pulse length. Accordingly, the plasma should be in a static equilibrium along the field lines. In the strongly collisional regimes typical for the edge plasma (see Sec. 2) one should expect only slow plasma flows along the field lines, with a considerable recycling of the neutrals. Therefore, we accept as a model for further analysis that of a pressure equilibrium along the field lines,

$$
p=2 n T=\text { const }
$$

Note that possible "wobbling" of the flux tube in the course of the dynamo activity does not affect this conclusion if the motions occur with a speed below the sound speed.

\subsection{The thermal equilibrium}

One can expect three heat sources for the edge plasma: thermal conduction from the plasma core, Joule heating by the parallel current, and visco-resistive dissipation of MHD perturbations driven by. possible MHD instability (note that a certain level of MHD fluctuations is required for helicity injection). To get some insights into the first mechanism, we assume that the cross-field diffusion coefficient and cross-field thermal diffusivity are some fraction $\alpha$ of the Bohm diffusion coefficient defined as

$$
D_{B}=(1 / 16)(c T / e B)
$$


Numerically,

$$
D_{B}\left(\mathrm{~cm}^{2} / \mathrm{s}\right)=6 \cdot 10^{2} T(\mathrm{eV}) / B(\mathrm{~T}) .
$$

Taking $T=20 \mathrm{eV}, B=2 \mathrm{~T}$, and $a=6 \mathrm{~cm}$, one finds that the cross-field diffusion time,

$$
\tau_{\perp-a^{2} / 2 \alpha D_{B}}
$$

is $3 \cdot 10^{-3} / \alpha$ (s). As we will see shortly, this time is much longer than the time of the parallel heat losses. Therefore, if the central column was supported by the cross-field heat transport, it would be necessarily hollow, with a considerable reduction of the axial current.

The plasma in the flux tubes that begin and end in the gun (or near the outer wall of the flux conserver) is considerably narrower than in the central column $(\sim 1 \mathrm{~cm})$. However, even there the cross-field diffusion time remains long, $\sim 10^{-4} / \alpha(\mathrm{s})$.

The thermal conductivity time to the walls can be evaluated as

$$
\tau_{\chi}-L^{2} / 8 \chi_{11}
$$

where $\chi_{11}$ is the parallel thermal diffusivity. In "practical" units,

$$
\chi_{11}\left(\mathrm{~cm}^{2} / \mathrm{s}\right)=8 \cdot 10^{19}\left[T_{e}(\mathrm{eV})\right]^{5 / 2} / Z_{e f f} n\left(\mathrm{~cm}^{-3}\right)
$$

For the set of parameters in $(5.1)$, and $L=200 \mathrm{~cm}$, one finds that $\tau_{\chi \sim 5} \cdot 10^{-6} \mathrm{~s}$, in other words, much shorter than the cross-field diffusion time.

The Joule heating provides a mechanism, which, in principle, can maintain plasma both in the central column and in the area near the outer wall. Let us define a characteristic Joule heating time as the time within which the thermal energy content is doubled by the Joule heating,

$$
\tau_{f}=(3 n T) /\left(j^{2} / \sigma\right)
$$

Numerically,

$$
\tau_{f}(\mathrm{~s})=6 \cdot 10^{-18} n(\mathrm{~cm})^{-3}[T(\mathrm{eV})]^{5 / 2} /\left[j\left(\mathrm{~A} / \mathrm{cm}^{2}\right)\right]^{2} Z_{\text {eff }}
$$

For the set of parameters in $(5.1)$, and $j \sim 1.5 \mathrm{kA} / \mathrm{cm}^{2}$, we find that $\tau_{j} \sim 3 \cdot 10^{-7} \mathrm{~s}$, by far shorter than the Bohm diffusion time even over a scale of a few $\mathrm{mm}$. So, probably, Joule heating is the main mechanism for sustaining the thermal equilibrium of the plasma on the open field lines.

Assuming that the main channel of the heat losses is parallel thermal conductivity, one can write the following balance equation: 


$$
\frac{\partial}{\partial s} \kappa_{11} \frac{\partial T}{\partial s}+\frac{j^{2}}{\sigma}=0
$$

where $\kappa_{l /}$ is electron thermal conductivity and $s$ is a distance along the field line. In Eq. (5.16), we assume that the magnetic field (and, therefore, the cross-section of the flux tube) do not vary along the field line. Assuming, for simplicity, the symmetry of the problem with respect to the equatorial plane, one finds the following relation for the plasma temperature in the equatorial plane:

$$
\frac{j^{2} L^{2}}{\sigma_{0} K_{\mathrm{M} 0} T_{0}}=\frac{8}{7} \int_{0}^{1} \frac{d z}{\sqrt{1-z^{4 / 7}}} \approx 1.6
$$

where the subscript " 0 " designates the values of the corresponding quantities in the equatorial plane. Eq. (5.17) yields the numerical estimate for the plasma temperature:

$$
T_{0}(\mathrm{eV})=0.25\left[j^{2}\left(\mathrm{~A} / \mathrm{cm}^{2}\right) L^{2}(\mathrm{~cm}) Z_{\text {eff }}^{2}\right]^{1 / 5}
$$

For $j=1.5 \mathrm{kA} / \mathrm{cm}^{2}, L=200 \mathrm{~cm}, Z_{\text {eff }}=2$, we find $T_{0}=30 \mathrm{eV}$. We see that the plasma temperature in this model is 1.5 times higher than in (5.1). At $T_{e}=30 \mathrm{eV}$, the thermal conduction time (5.12) and the Joule heating time (5.15) are of the same order (as they should be).

Near the wall, the temperature (as determined from Eq. (5.16)) varies according to the equation

$$
T \sim(\Delta s)^{2 n}
$$

where $\Delta s$ is a distance from the wall along the field line. The pressure equilibrium condition (5.8) then means that the density has a singularity at the wall, $n \sim(\Delta s)^{-2 /}$. This does not cause divergence of the total number of plasma particles (as the density singularity is integrable) but it may cause some increase of radiative losses (see Sec. 5.7).

The third energy source, the MHD activity, is difficult to quantify. We will return to the discussion of its possible role in Sec. 5.8.

\subsection{Radiative losses.}

At the temperature $\sim 20 \mathrm{eV}$ and the density $\sim 10^{14} \mathrm{~cm}^{-3}$, the plasma is highly ionized. Therefore, the line radiation of the neutral hydrogen does not play any role. In the temperature domain of concern to us the main contribution to radiative losses comes from the free-bound transitions. The radiation power per unit volume is:

$$
P_{\text {rad }}\left(\mathrm{W} / \mathrm{cm}^{3}\right)=2.3 \cdot 10^{-31} n^{2}\left(\mathrm{~cm}^{-3}\right) / T^{1 / 2}(\mathrm{eV})
$$


We define the time of radiative cooling $\tau_{\text {rad }}$ as

$$
\tau_{\text {rad }}=3 n T / P_{\text {rad. }}
$$

Equations (5.20) and (5.21) yield the following numerical estimate:

$$
\tau_{\text {rad }}(\mathrm{s})=2.1 \cdot 10^{12} T^{3 / 2}(\mathrm{eV}) / n\left(\mathrm{~cm}^{-3}\right)
$$

For plasma parameters as in (5.1), this time is very long, $\sim 0.5 \mathrm{~s}$. This means that radiative losses are relatively unimportant (see discussion of the role of impurities below).

Radiative losses increase (and $\tau_{\text {rad }}$ decreases) near the wall, where the temperature drops and the density grows. Using the scaling $T \sim(\Delta s)^{2 \pi}, n \sim(\Delta s)^{-2 n}$, one finds that $P_{\text {rad }} \sim(\Delta s)^{-5 n}$. In other words, the integral of $P_{r a d}$ over the field line converges near the wall and the main radiation losses occur from the region of higher temperature.

Equating (5.13) and (5.22), one finds the density at which Joule heating might become equal to radiative losses. At $Z_{\text {eff }}=2$, and $j=1.5 \mathrm{kA} / \mathrm{cm}^{2}$ this would happen at $n=10^{2} \mathrm{~cm}^{-3}$ (and at a corresponding very low temperature). The unreasonably high value of $n$ shows that, in fact, the radiative losses do not play any significant role in the edge plasma behavior.

Now we discuss the role of impurities. They affect the radiation loss of the edge plasma in two ways: first, they contribute to the free-bound radiation and, second, they are the source of the line radiation. To take into account the first contribution we should multiply expression (5.20) by approximately $Z_{e f f} Z^{2}$ where $Z$ is the average charge of the impurity ions. Assuming that, in a $20 \mathrm{eV}$ plasma, $Z=4$, we find that free-bound radiation increases by a factor of 32 in a plasma with $Z_{e f f}=2$. According to (5.22), this is insufficient to make the free-bound radiation an important process.

The power density of the line radiation can be evaluated as

$$
P_{\text {line }} \sim E_{\text {ex }}<\sigma_{e x} v>n n_{\mathrm{Z}}
$$

For a plasma with $T_{e} \sim 30 \mathrm{eV}$, levels with $E_{e x}$ up to $100-150 \mathrm{eV}$ will be excited. For these excitation energies the typical reactivity does not exceed $<\sigma_{e x} v>\sim 3 \cdot 10^{-8} \mathrm{~cm}^{3} / \mathrm{s}$. Accordingly, one has (for $\left.T_{e} \sim 20-30 \mathrm{eV}\right) P_{\text {line }}\left(\mathrm{eV}^{\mathrm{cm}} \mathrm{cm}^{3}\right) \sim 4.5 \cdot 10^{-6} \mathrm{n}\left(\mathrm{cm}^{-3}\right) \mathrm{n}_{\mathrm{z}}\left(\mathrm{cm}^{-3}\right)$. For the plasma temperature $T_{e}=30 \mathrm{eV}$, the radiation time

$$
\tau_{\text {line }}=3 n T / P_{\text {line }}
$$


is $2 \cdot 10^{7} / n_{Z}(\mathrm{~s})$. Even for an unrealistically high density of impurities, $n_{Z} \sim 3 \cdot 10^{13} \mathrm{~cm}^{-3}$, this time is longer than the Joule heating time (5.15). Accordingly, we conclude that radiation losses from the plasma edge are sub-dominant compared to the thermal conduction losses.

Note that the relative role of radiative losses in the plasma core may be much more important, because of the much higher plasma temperature and corresponding increase in the reactivity $\left\langle\sigma_{e x} v\right\rangle$, and because of the lower heating power (higher conductivity). Note also that the magnitude of line radiation from the edge plasma may be large enough to be a problem for diagnostics based on radiation from the core plasma.

\subsection{Plasma resistivity}

The current flowing along a certain flux tube determines resistive voltage drop $\Delta \mathrm{V}_{\text {res }}$ along the flux tube. The plasma resistivity scales as $T^{3 / 2}$; according to (5.19), near the wall the resistivity diverges as $(\Delta s)^{-3 n}$. However, this divergence is integrable and the main contribution to $\Delta \mathrm{V}_{\text {res }}$ comes from the central section of the flux tube, where the temperature is of the order of $T_{0}$. One has then:

$$
\Delta \mathrm{V}_{\text {res }} \sim j L / \sigma_{0}
$$

The numerical estimate for the plasma conductivity reads as:

$$
\sigma=1.2 \cdot 10^{13}[T(e V)]^{3 / 2} / Z_{\text {eff }}^{2}
$$

For $Z_{\text {eff }}=2, j=1.5 \mathrm{kA} \mathrm{cm}^{2}, L=2 \mathrm{~m}$, one finds from (21), (22) that

$$
\Delta \mathrm{V}_{\text {res }}(\mathrm{V}) \sim 4.5 \cdot 10^{4} / \mathrm{T}_{0}(\mathrm{eV}) \mathrm{J}^{3 / 2}
$$

For temperature below $15 \mathrm{eV}$, this voltage becomes higher than the voltage applied between the electrodes $(\sim 1 \mathrm{keV})$ and the current on the open field lines becomes small (or the applied voltage high). At higher temperatures, the Ohmic losses become relatively insignificant and the applied voltage is "consumed" by the dynamo activity. At a plasma temperature $\sim 30 \mathrm{eV}$ that is expected to be established at a current density $\sim 1.5 \mathrm{kA} / \mathrm{cm}^{2}$ (see Sec. 5.6), the resistive voltage is as low as $300 \mathrm{~V}$. Accordingly, to maintain the current on the open field lines at the nominal value, one might consider lowering of the driving voltage to $300 \mathrm{~V}$ during the steady-state phase of the discharge. Otherwise, one can expect an excessive growth of MHD activity and deterioration of core plasma confinement. Of course, one should remember that the estimates presented here are very crude and, in order to obtain reliable quantitative predictions, one will need to perform an extensive numerical 
analysis. Still, the capability of controlling the voltage pulse shape seems to be a desirable feature for the experiment.

\subsection{Heat flux through the separatrix}

To get some rough idea of how much energy is transported through the separatrix from the plasma core, we assume that the life-time of the core plasma is determined by the Bohm diffusion coefficient (9),

$$
\tau_{\text {core }} \sim r_{\text {cord }}^{2} 2 D_{\text {Bohm }}
$$

Assuming that $T_{\text {core }}=300 \mathrm{eV}, r_{\text {core }}=20 \mathrm{~cm}, B=2 \mathrm{~T}$, one finds that $\tau_{\text {core }}-2 \mathrm{~ms}$. Dividing the plasma energy content $3 n T\left(4 \pi r^{3}\right.$ cord $\left./ 3\right)$ by the confinement time, one can find the power flux through the separatrix. For $n=10^{14} \mathrm{~cm}^{-3}$, and other parameters as just mentioned, the energy content in the core is $450 \mathrm{~J}$, and the power flowing through the separatrix is $\sim 150$ $\mathrm{kW}$. Even if the core energy confinement time is a factor of 10 shorter, this is a relatively small fraction of the power supplied to the open field lines by the external voltage ( 1 $\mathrm{kV} \cdot 100 \mathrm{kA}=100 \mathrm{MW}$ ).

The previous discussion was directed to the experiment, in which the very high level of ohmic heating on the open field lines determines the power balance of the edge plasma. In a spheromak reactor, the power will probably be delivered directly to the core by alpha particles or by auxiallary current drive and heating systems. Then, the power flow from the plasma core will become the dominant factor determining the plasma properties in the edge plasma, as in tokamak reactors. A consequence is that there must be a narrow, relatively hot temperature scrape-off layer immediately adjacent to the closed flux-surface region, in order to have transport coefficients adequate to remove the power emerging from the core. As in a tokamak, the width of this SOL is determined by balancing radial and axial transport, and this width will impact the spread of deposition of the core heat flux on material walls.

\subsection{Particle balance (ionization equilibrium)}

The life-time of a deuterium atom in a plasma with respect to ionization is

$$
\tau_{i}=I / n<\sigma_{i} v>
$$

where the averaging of the electron impact ionization reactivity is performed over the electron distribution function. For $T \sim 300 \mathrm{eV}$ (i.e., for the core plasma), $\left\langle\sigma_{i} \mathrm{v}\right\rangle \sim 3 \cdot 10^{-8}$ 
$\mathrm{cm}^{3} / \mathrm{s}$ and, at $n \sim 10^{14} \mathrm{~cm}^{-3}$, the ionization time is $\sim 0.3 \mu \mathrm{s}$. This means that the plasma core is impermeable for the Franck-Condon $(\sim 1 \mathrm{eV})$ neutrals. Even charge-exchange neutrals formed on the plasma periphery and having energies $-20-30 \mathrm{eV}$, do not penetrate the core. In other words, the multiple charge exchange process does not make the core permeable for the neutrals formed at the plasma periphery. (Charge-exchange energy losses may, however, be important at lower plasma densities and high edge neutral densities. ${ }^{6}$ ) We conclude, therefore, that the whole confinement volume operates in a high-recycling mode, with the sources of ions situated on the plasma periphery (no sources in the core). Given the approximately uniform distribution of the plasma currents over the plasma volume, Ohmic heating also occurs predominantly at the plasma periphery (where the resistivity is higher). This may create a kind of flat temperature and density profile in the plasma core.

The plasma in the central column is also in the high recycling mode, with no particle sources acting near the equatorial plane. This justifies our assumption that the plasma there is in pressure equilibrium.

On the outer circumference of the plasma, the thickness of the plasma between the wall and the separatrix is small, $\sim 1-2 \mathrm{~cm}$. This part of the edge plasma is permeable to neutrals.

\subsection{Generation of impurities}

There are potentially two sources of impurities in the proposed experiment. The first is plasma contamination during the formation stage, when no closed flux surfaces have been formed and plasma is in direct contact with the electrodes in the gun. The amount of impurities trapped into the plasma at this stage can probably made relatively low: in past experiments with coaxial guns quite clean plasma could be produced.

The second source is generation of impurities on the walls of the flux conserver and their subsequent diffusion into the plasma core, across the magnetic field. Within the model of mechanical and thermal equilibrium along the field lines that we considered in Secs. $5.5,5.6$, with only cold plasma being in direct contact with the wall, the amount of impurities generated via sputtering of the wall, should be small. Direct penetration of neutral impurity atoms into the plasma core can be ruled out by the same arguments presented in Sec. 5.10. Those impurity atoms that get ionized near the wall may cause some problems, as they will be dragged by the thermal force along the field lines into the region of hotter plasmas and will be accumulated there. The penetration time can be estimated as $\left(L^{2} / 8\right) /\left[\lambda_{i m p} v_{i m p}\right]$ where $\lambda_{i m p}$ and $v_{i m p}$ are the scattering length and the thermal velocity of the impurity ions. Taking plasma parameters as in (5.1), for an impurity with 
$A=14$, and $L=2 \mathrm{~m}$, one finds that the penetration time is $\sim 3 \mathrm{~ms}$, somewhat longer than the expected pulse length. However, for a higher plasma temperature this time will become shorter than the pulse-length.

Let therefore assume that, at the separatrix, there is some finite concentration of impurities. Their further penetration into the plasma depends on the mechanism of the anomalous transport. If this is a convective transport caused by large-scale plasma motions, one can expect that the diffusion coefficient for the impurity ions will be the same as for the deuterons. Then, according to the comments made in Sec. 5.10, one can expect that the impurities will be distributed more or less uniformly over the plasma core, with approximately the same relative concentration as on the separatrix.

If transport is caused by microturbulence, then the diffusion coefficient for the impurities can be very different from that for the deuterium ions. Which is greater is impossible to say without getting into details of the transport process. It may happen that there exists an anomalous thermal force acting on the impurity ions, causing their accumulation in the hottest part of the plasma.

\subsection{Issues for further analysis.}

1. A study of the formation process (in the context of plasma behavior on the open field lines).

2. A more detailed analysis of the processes on the plasma-electrode interface.

3. Evaluation of the speed of impurity entrainment by the thermal force.

4. Extension of our analysis to the processes on the closed field lines just inside the separatrix.

5. A study of the effect of line-tying in the central part of the device on MHD perturbations in the paraxial area. (Experimental data on the effect on the tilt mode exists from the S-1 device. ${ }^{7}$ )

6. Analysis of the role of the divertor.

\section{Acknowledgments}

The authors are grateful to C. Barnes and T. Jarboe for their valuable comments.

\section{References}

1. S.I. Braginski. In: "Reviews of Plasma Physics", M.A. Leontovich, Ed, v.1, p. 205. Consultants Bureau, N-Y, 1965 
2. NRL Plasma Formulary. NRL Publication 0084-4040, Washington DC 20375-5000, 1987.

3. J. C. Fernandéz, et al., "Energy Confinement Studies in Spheromaks with Mesh Flux Conservers," Nucl. Fusion 28, 1555 (1988).

4. F. J. Wysocki, et al., "Improved Energy Confinement in Spheromaks with Reduced Field Errors," Phys. Rev. Letters 65, 40 (1990).

5. T. R. Jarboe and B. Alper, "Model for the loop voltage of reversed field pinches," Phys. Fluids 30, 1177 (1987).

6. R. M. Mayo, et al., "Effect of neutral particles on the energy confinement of spheromaks," Phys. Fluids B 2, 115 (1989).

7. F. J. Wysocki, "Experimental investigation of line-tying effects on the spheromak tilt mode," Phys. Fluids 30, 482 (1987). 


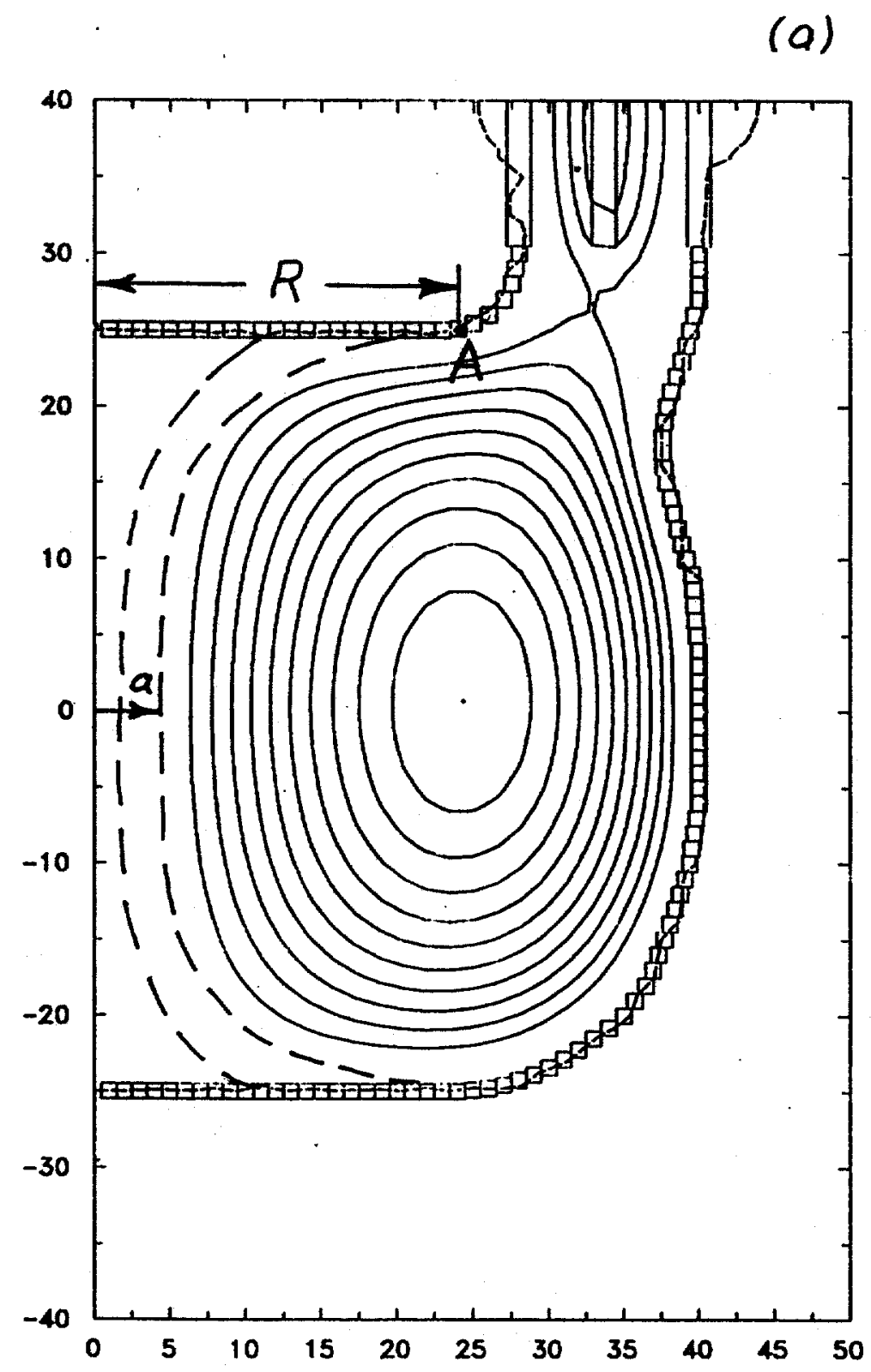

Figure 1. Magnetic field lines in the paraxial and edge regions.

(1a) Shown in dashed lines are field lines crossing the flux conserver because of the skin effect 


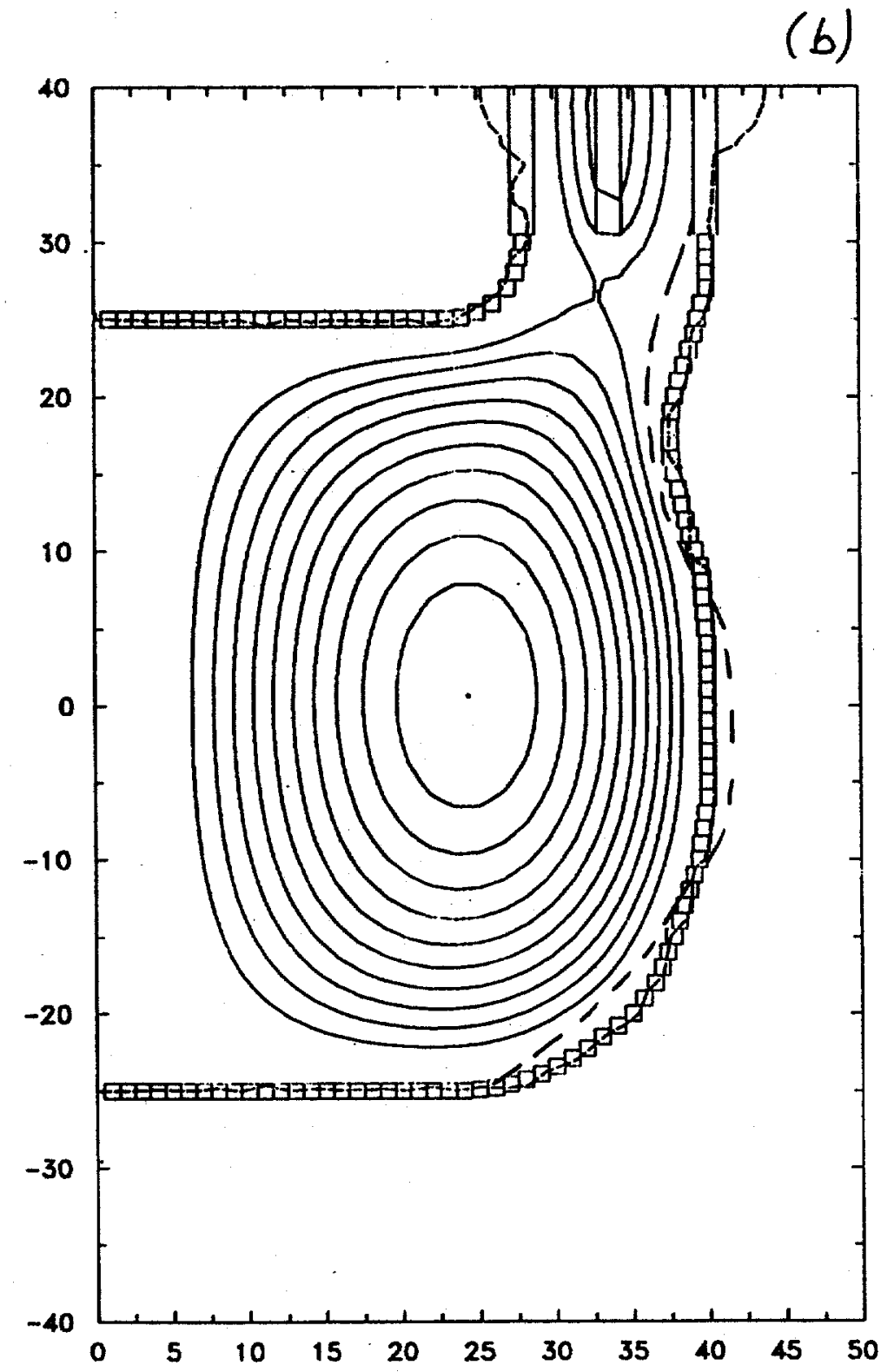

(1b) Because of magnetic field errors, "pockets" of cold plasma may be formed near the flux conserver (see right-hand-side of the figure) 


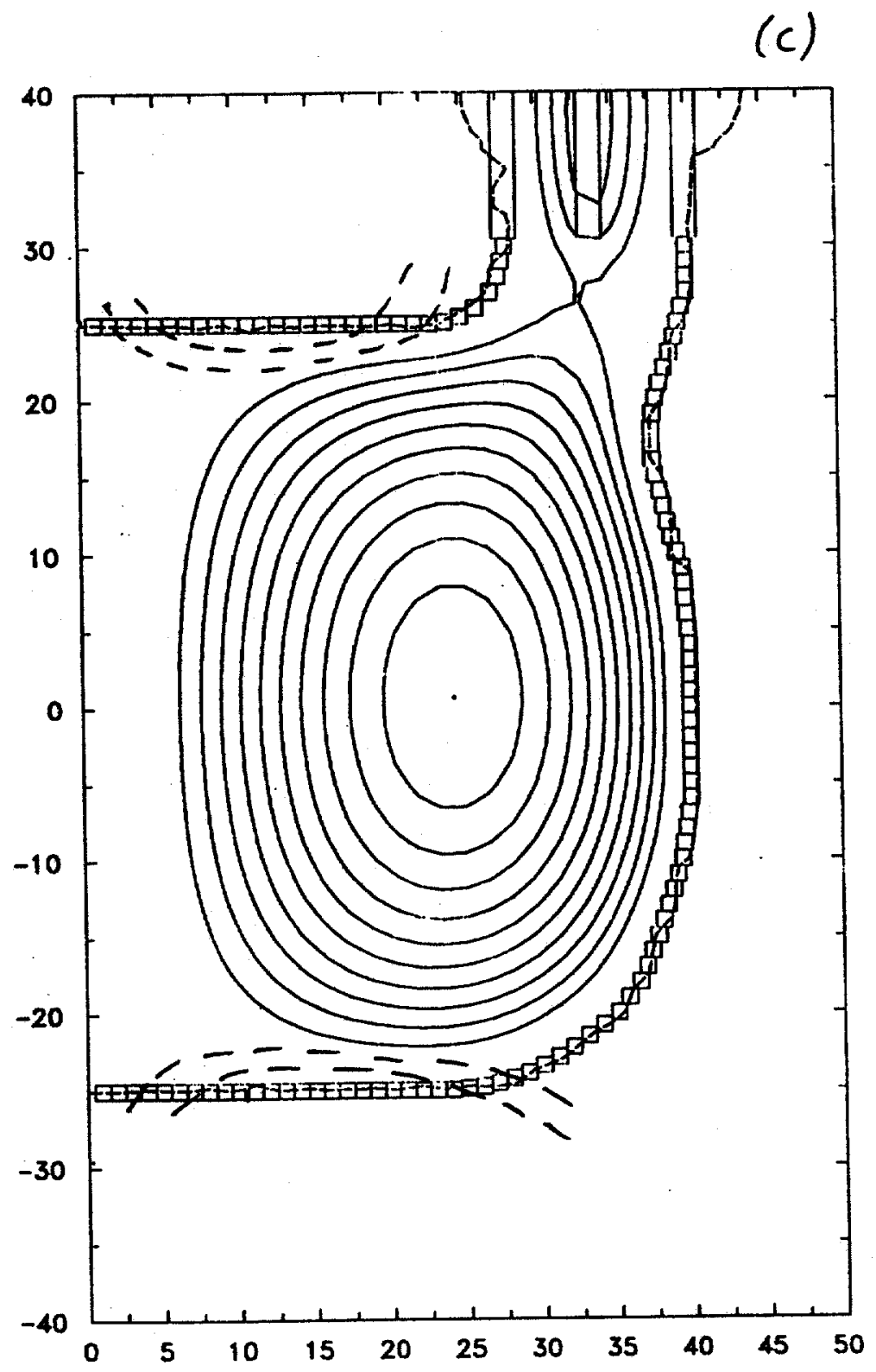

(1c) By introducing properly chosed bias magnetic field, one may magnetically insulate the major part of the upper and lower surfaces of the flux conserver 


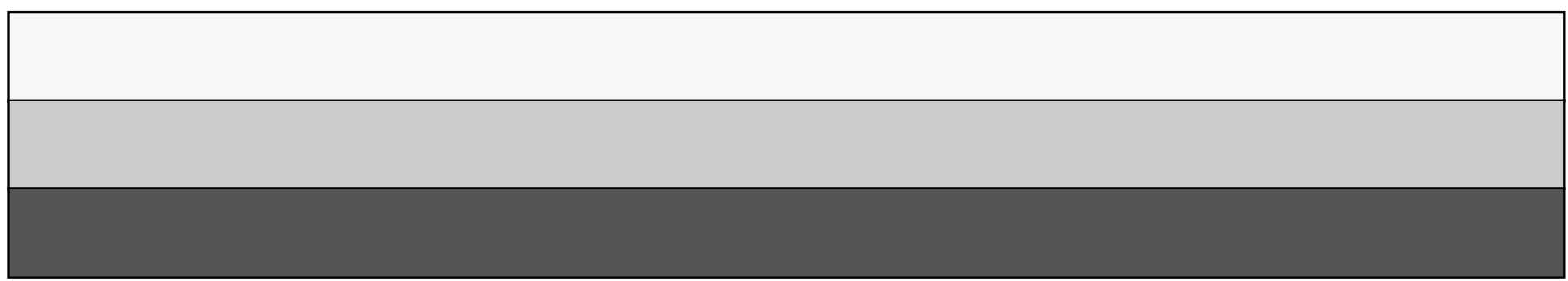

